\title{
ACTION OF HECKE OPERATORS ON MAASS THETA SERIES AND ZETA FUNCTIONS
}

\author{
A. N. ANDRIANOV
}

Dedicated to the centenary of the birth of Dmitriu Konstantinovich Faddeev

\begin{abstract}
The introductory part contains definitions and basic properties of harmonic theta series, Siegel modular forms, and Hecke operators. Then the transformation formulas are recalled, related to the action of modular substitutions and regular Hecke operators on general harmonic theta series, including specialization to the case of Maass theta series. The following new results are obtained: construction of infinite sequences of eigenfunctions for all regular Hecke operators on spaces of Maass theta series; in the case of Maass theta series of genus 2, all the eigenfunctions are constructed and the corresponding Andrianov zeta functions are expressed in the form of products of two $L$-functions of the relevant imaginary quadratic rings. The proofs are based on a combination of explicit formulas for the action of Hecke operators on theta series with Gauss composition of binary quadratic forms.
\end{abstract}

\section{INTRODUCTION}

The evident importance of the celebrated Shimura-Taniyama conjecture on the explicit relationship between Hasse zeta functions of elliptic curves and Hecke zeta functions of elliptic modular forms makes it quite natural to look for many-dimensional generalizations.

In 1980, Yoshida (11], p. 243) stated a two-dimensional analog of the ShimuraTaniyama conjecture. It asserts that the zeta function of a two-dimensional Abelian variety over the field of rational numbers is (up to a finite number of Euler factors) the (Andrianov) zeta function of a cusp form of weight 2 for a congruence subgroup of the Siegel modular group of genus 2. Yoshida also gave an example supporting the conjecture. In 1993, R. Salvati-Manni and J. Top [10] considered other interesting examples that corroborate the Yosida conjecture.

In view of possible relationships between zeta functions of algebraic varieties and zeta functions of modular forms, it would be of interest to compute more zeta functions of specific modular forms. The zeta functions of modular forms are defined as certain Euler products associated with common eigenfunctions of sufficiently complete families of Hecke operators. The most common sources for the search of eigenfunctions are given by spaces of theta series of integral positive definite quadratic forms weighted by harmonic polynomials. General formulas for the modular transformation of harmonic theta series and for their transformation under Hecke operators were obtained in our papers 5 and [6], respectively, and in $\S \S 1-3$ we briefly recall the transformation formulas in a somewhat modified form.

2000 Mathematics Subject Classification. Primary 11F27, 11F46, 11F60, 11F66.

Key words and phrases. Harmonic theta series, Hecke operators, Maass theta series, Siegel modular forms, zeta functions of Siegel modular forms.

Supported in part by RFBR (grant no. 05-01-00930). 
In 1977, H. Maass [9] introduced an important class of harmonic theta series of the form

$$
\Theta_{\nu}(Z, Q, \chi)=\sum_{N \in \mathbb{Z}_{m}^{m}} \chi(\operatorname{det} N)(\operatorname{det} N)^{\nu} \exp \left(\pi i \operatorname{Trace}\left({ }^{t} N Q N Z\right)\right),
$$

where $Z$ belongs to the Siegel upper half-plane of genus $m, Q$ is the matrix of an integral positive definite quadratic form in $m$ variables, $\chi$ is a Dirichlet character, and $\nu=0,1$ (if $\nu=0$, it is assumed that $\chi(\operatorname{det} N)(\operatorname{det} N)^{\nu}=\chi(\operatorname{det} N)$ for all $\left.N\right)$. In the same paper Maass established automorphic properties of the series and indicated a number of cases where the series are not identically zero. He also suggested that "die Thetareihen (*) in der von Andrianov [1] begründeten Theorie der Dirichletreihen zu Modulformen zweiten Grades und ihrer Verallgemeinerung eine wichtige Rolle spilen werden". At that time I did not pay much attention to the remark; moreover, the action of Hecke operators on theta series had not yet been investigated. Now I can say that Maass was right and can provide some positive evidence. In $\S 3$ (Proposition 7 ) we prove that if the proper class number of $Q$ is 1 , then the Maass series is an eigenfunction for all regular Hecke operators, for every character $\chi$. Theorem 12 of $\S 4$ establishes that for $m=2$ linear combinations of the Maass series with characters of the group of proper classes of binary quadratic forms (with respect to the Gauss composition) are eigenfunctions for all regular Hecke operators with explicitly given eigenvalues. The proof is based on a combination of explicit formulas for the action of Hecke operators on harmonic theta series and the technique introduced in 1 and relating Hecke operators on modular forms of genus 2 to Gauss composition of binary quadratic forms. This result allows us to show that the (Andrianov) zeta function of each eigenfunction of this sort is the product of two $L$-functions of the corresponding imaginary quadratic ring ( $\S 5$, Theorem 15 ).

Notation. We reserve the letters $\mathbb{Z}, \mathbb{Q}, \mathbb{R}$, and $\mathbb{C}$ for the ring of rational integers, the field of rational numbers, the field of real numbers, and the field of complex numbers, respectively. $\mathbb{A}_{n}^{m}$ is the set of all $(m \times n)$-matrices with entries in a set $\mathbb{A}$.

If $M$ is a matrix, ${ }^{t} M$ always denotes the transpose of $M$. If the entries of $M$ belong to $\mathbb{C}$, then $\bar{M}$ is the matrix with complex conjugate entries. $1_{n}$ and $0_{n}$ denote the unit matrix and the zero matrix of order $n$, respectively.

For a symmetric matrix $Q$, we write

$$
Q[M]={ }^{t} M Q M
$$

if the product on the right is defined.

We denote by

$$
\mathbb{E}^{m}=\left\{Q=\left(q_{\alpha \beta}\right) \in \mathbb{Z}_{m}^{m} \mid{ }^{t} Q=Q, q_{11}, q_{22}, \ldots, q_{m m} \in 2 \mathbb{Z}\right\}
$$

the set of all even matrices of order $m$, i.e., the set of matrices of integral quadratic forms

$$
\mathbf{q}(X)=\frac{1}{2} Q[X]=\frac{1}{2}{ }^{t} X Q X \quad\left({ }^{t} X=\left(x_{1}, \ldots, x_{m}\right)\right)
$$

in $m$ variables. We recall that the level $q$ of an invertible matrix $Q \in \mathbb{E}^{m}$ (and of the corresponding form) is the smallest positive integer satisfying $q Q^{-1} \in \mathbb{E}^{m}$, and the divisor $d$ of a nonzero $Q \in \mathbb{E}^{m}$ (and that of the corresponding form) is the greatest positive integer such that $d^{-1} Q \in \mathbb{E}^{m}$; the matrices and forms of the divisor 1 are called primitive. 


\section{$\S 1$. Harmonic theta series and MaAss theta series}

Here we recall the basic definitions and properties of harmonic theta series of integral positive definite quadratic forms. For the details and proofs, see [5], [7], or [8].

Let $Q \in \mathbb{E}^{m}$ be the matrix of an integral positive definite quadratic form $\mathbf{q}$ in $m$ variables. For $n \in \mathbb{N}$, let $P$ be a polynomial on $\mathbb{C}_{n}^{m}$. Suppose that the polynomial is homogeneous of exponent $\nu$ and harmonic with respect to the quadratic form $\mathbf{q}$. These conditions mean that $P$ satisfies

$$
P(V A)=(\operatorname{det} A)^{\nu} P(V) \quad\left(\forall V \in \mathbb{C}_{n}^{m} \text { and } A \in G L_{n}(\mathbb{C})\right)
$$

and has the form $P(V)=P_{0}(S V)$, where $S$ is a real $(n \times n)$-matrix such that $Q={ }^{t} S S$, and the polynomial $P_{0}$ satisfies the Laplace equation

$$
\Delta P_{0}=\sum_{\alpha, \beta} \frac{\partial^{2} P_{0}(V)}{\left(\partial v_{\alpha \beta}\right)^{2}}=0 .
$$

The theta series of genus $n$ of the matrix $Q$ with the coefficient polynomial $P$ and characteristic

$$
L \in L_{n}(Q)=\left\{L \in \mathbb{Z}_{n}^{m} / q \mathbb{Z}_{n}^{m} \mid Q L \equiv 0(\bmod q)\right\}
$$

where $q$ is the level of $Q$, is defined by

$$
\Theta_{P}(Z, Q \mid L)=\sum_{N \in \mathbb{Z}_{n}^{m}} P\left(N+q^{-1} L\right) e\left\{Q\left[N+q^{-1} L\right] Z\right\},
$$

where $Z$ belongs to the upper half-plane of genus $n$,

$$
Z \in \mathbb{H}^{n}=\left\{Z=X+\sqrt{-1} Y \in \mathbb{C}_{n}^{n} \mid{ }^{t} Z=Z, Y>0\right\}
$$

and, for a square matrix $A$, we write

$$
e\{A\}=e^{\pi \sqrt{-1} \operatorname{Trace} A}
$$

The theta series converge absolutely and uniformly on compact subsets of $\mathbb{H}^{n}$. If $U$ is an integral unimodular matrix of order $m$, then, since $U \mathbb{Z}_{n}^{m}=\mathbb{Z}_{n}^{m}$, the replacement of $N$ with $U N$ in (1.1) leads to the identities

$$
\begin{aligned}
\Theta_{P}(Z, Q \mid L) & =\sum_{N \in \mathbb{Z}_{n}^{m}} P\left(U\left(N+q^{-1} U^{-1} L\right)\right) e\left\{Q\left[U\left(N+q^{-1} U^{-1} L\right)\right] Z\right\} \\
& =\Theta_{P \mid U}\left(Z, Q[U] \mid U^{-1} L\right) \quad \forall U \in G L_{m}(\mathbb{Z}),
\end{aligned}
$$

where $(P \mid U)(V)=P(U V)$.

Examples. 1. Clearly, the constant polynomial $P(V)=1$ is a harmonic polynomial of exponent $\nu=0$ with respect to every quadratic form $\mathbf{q}$, and the theta series (1.1) turns into the usual theta series

$$
\Theta_{0}(Z, Q \mid L)=\sum_{N \in \mathbb{Z}_{n}^{m}} e\left\{Q\left[N+q^{-1} L\right] Z\right\} \quad\left(Z \in \mathbb{H}^{n}, L \in L_{n}(Q)\right)
$$

of genus $n$ of the form $\mathbf{q}$ (or matrix $Q$ ) with characteristic $L$.

2 . If $n=m$, the polynomial

$$
P(V)=\operatorname{det} V \quad\left(V=\left(v_{\alpha \beta}\right) \in \mathbb{C}_{m}^{m}\right)
$$

is homogeneous of exponent 1 and is linear in each of the $m^{2}$ variables $v_{\alpha \beta}$. It follows that the polynomial $P_{0}(T)=P\left(S^{-1} T\right)$ is also linear in each of the variables for every nonsingular $(m \times m)$-matrix $S$, and so it satisfies the Laplace equation. Thus, this polynomial is homogeneous of exponent 1 and harmonic with respect to every positive 
definite quadratic form $\mathbf{q}(X)$ in $m$ variables. The corresponding theta series of genus $m$ looks like this:

$$
\Theta_{1}(Z, Q \mid L)=\sum_{N \in \mathbb{Z}_{m}^{m}} \operatorname{det}\left(N+q^{-1} L\right) e\left\{Q\left[N+q^{-1} L\right] Z\right\} \quad\left(Z \in \mathbb{H}^{m}, L \in L_{m}(Q)\right) .
$$

3. The Maass theta series (*) are linear combinations of series (1.5) with $n=m$ or series (1.6), depending on whether $\nu$ is equal to 0 or to 1 : if $\chi$ is a character modulo $g$, then

$$
\begin{aligned}
& \Theta_{\nu}(Z, Q, \chi) \\
& \quad=\sum_{R \in \mathbb{Z}_{m}^{m} / g \mathbb{Z}_{m}^{m}} \chi(\operatorname{det} R) g^{\nu m} \sum_{N \in \mathbb{Z}_{m}^{m}} \operatorname{det}^{\nu}\left(N+\frac{q g}{q g^{2}} R\right) e\left\{g^{2} Q\left[N+\frac{q g}{q g^{2}} R\right] Z\right\} \\
& \quad=g^{\nu m} \sum_{R \in \mathbb{Z}_{m}^{m} / g \mathbb{Z}_{m}^{m}} \chi(\operatorname{det} R) \Theta_{\nu}\left(Z, g^{2} Q \mid q g R\right),
\end{aligned}
$$

where $q$ is the level of $Q$ (note that the level of $g^{2} Q$ is $q g^{2}$, and for every $R \in \mathbb{Z}_{m}^{m}$ we have $\left.g^{2} Q(q g R) \equiv 0\left(\bmod q g^{2}\right)\right)$.

The following theorem is a particular case of the well-known transformation formulas for harmonic theta functions (see, e.g., [5, Theorems 4.1-4.3]). In order to formulate the theorem, it is convenient to view the theta series $\Theta_{P}(Z, Q \mid L)$ with various characteristics $L \in L_{n}(Q)$ as entries of the theta column

$$
\boldsymbol{\Theta}_{P}(Z, Q)=\left(\begin{array}{c}
\vdots \\
\Theta_{P}\left(Z, Q \mid L_{\alpha}\right) \\
\vdots
\end{array}\right)
$$

of order

$$
l_{n}(Q)=\#\left(L_{n}(Q)\right)^{L \rightarrow q \underline{\underline{Q}}^{-1} L^{\prime}} \#\left(\mathbb{Z}_{n}^{m} / Q \mathbb{Z}_{n}^{m}\right)=(\operatorname{det} Q)^{n},
$$

where we assume that all different residue classes of $L_{n}(Q)$ are ordered in a fixed way.

Theorem 1. Let $Q$ be an even positive definite matrix of order $m$, and let $P$ be a homogeneous polynomial on $\mathbb{C}_{n}^{m}$ of an integral nonnegative exponent $\nu$ and harmonic with respect to the quadratic form with matrix $Q$. Then the following assertions are true.

1) For every integral symplectic matrix

$$
M=\left(\begin{array}{ll}
A & B \\
C & D
\end{array}\right) \in \Gamma^{n}=S p_{n}(\mathbb{Z})=\left\{M \in \mathbb{Z}_{2 n}^{2 n} \mid{ }^{t} M J_{n} M=J_{n}\right\},
$$

where

$$
J_{n}=\left(\begin{array}{cc}
0_{n} & 1_{n} \\
-1_{n} & 0_{n}
\end{array}\right)
$$

there is a square matrix $\Lambda_{Q}(M)$ of order $l_{n}(Q)$, independent of $Z$, such that the theta column satisfies the functional equation

$$
\boldsymbol{\Theta}_{P}\left((A Z+B)(C Z+D)^{-1}, Q\right)=\operatorname{det}(C Z+D)^{\nu+m / 2} \Lambda_{Q}(M) \boldsymbol{\Theta}_{P}(Z, Q) .
$$

If the order $m$ is even, then the matrices $\Lambda_{Q}(M)$ satisfy the relations

$$
\Lambda_{Q}\left(M M^{\prime}\right)=\Lambda_{Q}(M) \Lambda_{Q}\left(M^{\prime}\right) \quad\left(M, M^{\prime} \in \Gamma^{n}\right) .
$$

2) Suppose that

$$
M=\left(\begin{array}{cc}
A & B \\
C & D
\end{array}\right) \in \Gamma_{0}^{n}(q)=\left\{\left(\begin{array}{cc}
A & B \\
C & D
\end{array}\right) \in \Gamma^{n} \mid C \equiv 0_{n}(\bmod q)\right\},
$$


where $q$ is the level of $Q$. Then the entries $\Lambda_{Q}(M)_{\alpha \beta}$ of the matrix $\Lambda_{Q}(M)$ can be defined by the formulas

$$
\Lambda_{Q}(M)_{\alpha \beta}= \begin{cases}\mu_{Q}(M) e\left\{q^{-2} Q\left[L_{\beta}\right]^{t} D B\right\} & \text { if } L_{\beta} \equiv L_{\alpha} A \quad(\bmod q), \\ 0 & \text { otherwise, }\end{cases}
$$

where $\mu_{Q}(M)$ is an eighth root of unity.

3) If the level $q$ of $Q$ is equal to 1 , then the order $m$ of $Q$ is divisible by 8 , and

$$
\mu_{Q}(M)=1 \quad\left(\forall M \in \Gamma^{n}\right),
$$

but if $q>1$ and $m$ is even, then for every $M=\left(\begin{array}{cc}A & B \\ C & D\end{array}\right) \in \Gamma_{0}^{n}(q)$ the scalar $\mu_{Q}(M)$ can be computed by the formula

$$
\mu_{Q}(M)=\chi_{Q}(\operatorname{det} D)
$$

where $\chi_{Q}$ is the real Dirichlet character modulo $q$ satisfying

$$
\begin{aligned}
\chi_{Q}(-1) & =(-1)^{m / 2}, \\
\chi_{Q}(p) & =\left(\frac{(-1)^{m / 2} \operatorname{det} Q}{p}\right) \quad(\text { the Legendre symbol })
\end{aligned}
$$

if $p$ is an odd prime number that does not divide $q$, and

$$
\chi_{Q}(2)=2^{-m / 2} \sum_{r \in \mathbb{Z}^{m} / 2 \mathbb{Z}^{m}} e^{\frac{\pi i}{2} Q[r]}
$$

if $q$ is odd.

In principle, when $m$ is even, relations (1.9) make it possible to evaluate the multiplier matrices $\Lambda_{Q}(M)$ for all $M \in \Gamma^{n}$ if they are known for a system of generators of $\Gamma^{n}$. It is well known that the matrices

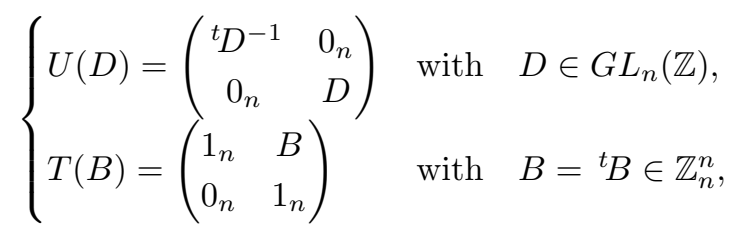

together with the matrix $J_{n}$, generate the group $\Gamma^{n}$ (see, e.g., [2, Theorem 1.3.6]). By part 2) of Theorem 1 or by direct computation, we obtain

$$
\Theta_{P}\left({ }^{t} D^{-1} Z D^{-1}, Q \mid L\right)=\operatorname{det}(D)^{\nu+m / 2} \chi_{Q}(\operatorname{det} D) \Theta_{P}\left(Z, Q \mid L^{t} D^{-1}\right)
$$

and

$$
\Theta_{P}(Z+B, Q \mid L)=e\left\{q^{-2} Q[L] B\right\} \Theta_{P}(Z, Q \mid L),
$$

where $L \in L_{n}(Q), D \in G L_{n}(\mathbb{Z})$, and $B={ }^{t} B \in \mathbb{Z}_{n}^{n}$. As to the generator $J_{n}$, the inversion formulas for harmonic theta functions (see [5, Lemma 5.1]) give the formula

$$
\Theta_{P}\left(-Z^{-1}, Q \mid L\right)=\operatorname{det}(-Z)^{\nu+m / 2} \frac{i^{m n / 2}}{\sqrt{(\operatorname{det} Q)^{n}}} \sum_{L^{\prime} \in L_{n}(Q)} e\left\{2 q^{-2 t} L Q L^{\prime}\right\} \Theta_{P}\left(Z, Q \mid L^{\prime}\right)
$$

for $L \in L_{n}(Q)$. 


\section{§2. MOdUlar FORMS}

Here we recall the basic definitions and properties of Siegel modular forms of integral weights. The general real positive symplectic group of genus $n$, consisting of all real symplectic matrices of order $2 n$ with positive multipliers,

$$
\mathbb{G}^{n}=G S p_{n}^{+}(\mathbb{R})=\left\{M \in \mathbb{R}_{2 n}^{2 n} \mid{ }^{t} M J_{n} M=\mu(M) J_{n}, \mu(M)>0\right\},
$$

is a real Lie group acting as a group of analytic automorphisms on the $n(n+1) / 2$ dimensional open complex variety (1.2) by the rule

$$
\mathbb{G}^{n} \ni M=\left(\begin{array}{ll}
A & B \\
C & D
\end{array}\right): Z \mapsto M\langle Z\rangle=(A Z+B)(C Z+D)^{-1} \quad\left(Z \in \mathbb{H}^{n}\right) .
$$

Acting on the upper half-plane $\mathbb{H}^{n}$, the general symplectic group operates also on complex-valued functions $F$ on $\mathbb{H}^{n}$ by Petersson operators of integral weights $k$,

$$
\mathbb{G}^{n} \ni M=\left(\begin{array}{ll}
A & B \\
C & D
\end{array}\right):\left.F \mapsto F\right|_{k} M=\operatorname{det}(C Z+D)^{-k} F(M\langle Z\rangle) .
$$

The Petersson operators obey the rules

$$
\left.F\right|_{k} M M^{\prime}=\left.\left(\left.F\right|_{k} M\right)\right|_{k} M^{\prime} \quad\left(M, M^{\prime} \in \mathbb{G}^{n}\right) .
$$

Let $S$ be a subgroup of $\mathbb{G}^{n}$ commensurable with the modular group $\Gamma^{n}$, let $\chi$ be a character of $S$, that is, a multiplicative homomorphism of $S$ into the nonzero complex numbers with kernel of finite index in $S$, and let $k$ be an integer. A complex-valued function $F$ on $\mathbb{H}^{n}$ is called a (Siegel) modular form of weight $k$ and character $\chi$ for the group $S$ if the following conditions are fulfilled: (i) $F$ is a holomorphic function in $n(n+1) / 2$ complex variables on $\mathbb{H}^{n}$; (ii) for every matrix $M \in S$, the function $F$ satisfies the functional equation

$$
\left.F\right|_{k} M=\chi(M) F,
$$

where $\left.\right|_{k}$ is the Petersson operator of weight $k$; (iii) if $n=1$, then every function $\left.F\right|_{k} M$ with $M \in \Gamma^{1}$ is bounded on each subset of $\mathbb{H}^{1}$ of the form $\mathbb{H}_{\varepsilon}^{1}=\left\{x+i y \in \mathbb{H}^{1} \mid y \geq \varepsilon\right\}$ with $\varepsilon>0$.

Clearly, the set $\mathfrak{M}_{k}(S, \chi)$ of all modular forms of weight $k$ and character $\chi$ for the group $S$ is a linear space over the field $\mathbb{C}$. Each space $\mathfrak{M}_{k}(S, \chi)$ has finite dimension over $\mathbb{C}$.

For a positive integer $q$, we denote by

$$
\Gamma^{n}(q)=\left\{M \in \Gamma^{n} \mid M \equiv 1_{2 n}(\bmod q)\right\}
$$

the principal congruence subgroup of level $q$ of the modular group. $\Gamma^{n}(q)$ is a normal subgroup of finite index in the modular group.

A subgroup $S$ of the (real) symplectic group

$$
\mathbb{S}^{n}=\left\{M \in \mathbb{G}^{n} \mid \mu(M)=1\right\}
$$

is called a congruence subgroup if it contains a principal congruence subgroup as a subgroup of finite index. A character of such an $S$ is said to be a congruence character if it is trivial on a principal congruence subgroup contained in $S$. If $S$ is a congruence subgroup and $\chi$ is a congruence character of $S$, then, for each modular form $F$ of $\mathfrak{M}_{k}(S, \chi)$ and each matrix $M \in \mathbb{G}^{n} \cap \mathbb{Q}_{2 n}^{2 n}$, the function $\left.F\right|_{k} M$ has a Fourier expansion of the form

$$
\left(\left.F\right|_{k} M\right)(Z)=\sum_{A \in \mathbb{E}^{n}, A \geq 0} f_{M}(A) e\{A Z / r\}
$$


with a positive integer $r$ depending on $S$, $\chi$, and $M$, which converges absolutely on $\mathbb{H}^{n}$ and uniformly on every subset of the form

$$
\mathbb{H}_{\varepsilon}^{n}=\left\{Z=X+i Y \in \mathbb{H}^{n} \mid Y \geq \varepsilon 1_{2 n}\right\} \quad \text { with } \quad \varepsilon>0 .
$$

The modular form $F$ is called a cusp form if the coefficients $f_{M}(A)$ of the expansion (2.5) satisfy the conditions

$$
f_{M}(A)=0 \quad \text { for all } M \in \Gamma^{n} \text { and } A \in \mathbb{E}^{n} \text { with } \operatorname{det} A=0 .
$$

The subspace of cusp forms of $\mathfrak{M}_{k}(S, \chi)$ will be denoted by $\mathfrak{N}_{k}(S, \chi)$.

Theorem 2. Let $Q$ be an even positive definite matrix of an even order $m$, and let $P$ be a homogeneous polynomial on $\mathbb{C}_{n}^{m}$ of an integral nonnegative exponent $\nu$ and harmonic with respect to the quadratic form with matrix $Q$. Then each of the theta series of genus $n$ of the matrix $Q$ with the coefficient form $P$ and characteristic $L \in L_{n}(Q)$ is a modular form of weight $k=\nu+m / 2$ and the unit character for the group $\Gamma^{n}(q)$, where $q$ is the level of $Q$ :

$$
\Theta_{P}(Z, Q \mid L) \in \mathfrak{M}_{\nu+m / 2}\left(\Gamma^{n}(q), 1\right)=\mathfrak{M}_{\nu+m / 2}^{n}(q) .
$$

If $\nu>0$, then the theta series is a cusp form:

$$
\Theta_{P}(Z, Q \mid L) \in \mathfrak{N}_{\nu+m / 2}\left(\Gamma^{n}(q), 1\right)=\mathfrak{N}_{\nu+m / 2}^{n}(q) \quad(\nu>0) .
$$

Proof. Trivial estimates show that each of the theta series (1.1) converges absolutely on $\mathbb{H}^{n}$ and uniformly on the subsets indicated in (2.6). It follows that each of these theta series satisfies condition (i) and, by part 1) of Theorem 1, condition (iii) (see the definition of modular forms). By Theorem 1, the multiplier matrix $\Lambda_{Q}(M)$ is the unit matrix of order $l_{n}(Q)$ if $M \in \Gamma^{n}(q)$. Thus, each function $F(Z)=\Theta_{P}(Z, Q \mid L)$ satisfies

$$
\left(\left.F\right|_{k} M\right)(Z)=\operatorname{det}(C Z+D)^{-k} F(M\langle Z\rangle)=F(Z) \quad \text { for every } \quad M=\left(\begin{array}{ll}
A & B \\
C & D
\end{array}\right) \in \Gamma^{n}(q),
$$

so that it satisfies condition (ii) for $S=\Gamma^{n}(q)$ with $\chi=1$.

In order to prove (2.8), first we note that the condition $\nu>0$ implies that $P(T)=0$ for every matrix $T \in \mathbb{C}_{n}^{m}$ with $\operatorname{rank}(T)<n$. Indeed, in this case there is a nonzero complex $n$-column $\mathbf{b}$ such that $T \mathbf{b}=0$. Let $B$ be a nonsingular square matrix with the first column $\mathbf{b}$. Then the matrix $T B$ has zero first column, whence $T B A=T B$ for every matrix $A$ of the form $\left(\begin{array}{cc}a & \mathbf{0} \\ t_{0} & 1_{n-1}\end{array}\right)$ with arbitrary $a \in \mathbb{C}$. It follows that

$$
P(T B A)=(\operatorname{det} A)^{\nu} P(T B)=a^{\nu} P(T B)=P(T B) .
$$

Thus, $P(T B)=0$, whence $P(T)=(\operatorname{det} B)^{-\nu} P(T B)=0$. Now, obviously, the Fourier expansion of the series (1.1) can be written in the form

$$
\Theta_{P}(Z, Q \mid L)=\sum_{A \in \mathbb{E}^{n}, A \geq 0}\left(\sum_{\substack{N \in \mathbb{Z}_{-}^{m}, q Q\left[N+q^{-1} L\right]=A}} P\left(N+q^{-1} L\right)\right) e\{A Z / q\} .
$$

Since $Q>0$, the condition $\operatorname{det} A=\operatorname{det}\left(q Q\left[N+q^{-1} L\right]\right)=0$ implies that $\operatorname{rank}\left(N+q^{-1} L\right)<$ $n$, and, by the above, we have $P\left(N+q^{-1} L\right)=0$, so that the corresponding Fourier coefficient of the series (2.9) is equal to zero. Then, using part 1) of Theorem 1, we conclude that for every $M \in \Gamma^{n}$ the function $\left.\Theta_{P}(Z, Q \mid L)\right|_{k} M$ is a linear combination with constant coefficients of the series $\Theta_{P}\left(Z, Q \mid L_{\alpha}\right)$, and so, together with these series, has zero Fourier coefficients corresponding to the singular arguments.

In the case of Maass theta series $(*)$, we can say more. 
Proposition 3 (Maass, 1977). Let $Q$ be an even positive definite matrix of even order $m, g$ a positive integer, and $\chi$ a Dirichlet character modulo $g$. Then the Maass theta series $(*)$ are modular forms of weight $\nu+m / 2$ for the group $\Gamma_{0}^{m}\left(q g^{2}\right)$, with the character $\chi \chi_{Q}$ given by

$$
\begin{array}{r}
\Gamma_{0}^{m}\left(q g^{2}\right) \ni M=\left(\begin{array}{cc}
A & B \\
C & D
\end{array}\right) \mapsto\left(\chi \chi_{Q}\right)(M)=\chi(\operatorname{det} D) \chi_{Q}(\operatorname{det} D): \\
\Theta_{\nu}(Z, Q, \chi) \in \mathfrak{M}_{\nu+m / 2}\left(\Gamma_{0}^{m}\left(q g^{2}\right), \chi \chi_{Q}\right) \quad(\nu=0,1),
\end{array}
$$

where $q$ is the level of $Q$ and $\chi_{Q}$ the character of $Q$ defined in Theorem 1.

If $\nu=1$, then the series $(*)$ is a cusp form:

$$
\Theta_{1}(Z, Q, \chi) \in \mathfrak{N}_{1+m / 2}\left(\Gamma_{0}^{m}\left(q g^{2}\right), \chi \chi_{Q}\right) .
$$

Proof. Since the level of $g^{2} Q$ is $q g^{2}$, application of Theorem 1 for a matrix $M=\left(\begin{array}{ll}A & B \\ C & D\end{array}\right)$ of $\Gamma_{0}^{m}\left(q g^{2}\right)$ to the theta series on the right-hand side of (1.7) yields

$$
\begin{aligned}
\left.\Theta_{\nu}\left(Z, g^{2} Q \mid q g R\right)\right|_{\nu+m / 2} M & =\chi_{g^{2} Q}(\operatorname{det} D) e\left\{\left(q g^{2}\right)^{-2}\left(g^{2} Q\right)[q g R A]^{t} D B\right\} \Theta_{\nu}\left(Z, g^{2} Q \mid q g R A\right) \\
& =\chi_{Q}(\operatorname{det} D) \Theta_{\nu}\left(Z, g^{2} Q \mid q g R A\right) .
\end{aligned}
$$

Hence, since $A^{t} D \equiv 1_{m}\left(\bmod q g^{2}\right)$, we get

$$
\begin{aligned}
\left.\Theta_{\nu}(Z, Q, \chi)\right|_{\nu+m / 2} M & =\left.g^{\nu m} \sum_{R \in \mathbb{Z}_{m}^{m} / g \mathbb{Z}_{m}^{m}} \chi(\operatorname{det} R) \Theta_{\nu}\left(Z, g^{2} Q \mid q g R\right)\right|_{\nu+m / 2} M \\
& =\chi_{Q}(\operatorname{det} D) g^{\nu m} \sum_{R \in \mathbb{Z}_{m}^{m} / g \mathbb{Z}_{m}^{m}} \chi\left(\operatorname{det} R A^{t} D\right) \Theta_{\nu}\left(Z, g^{2} Q \mid q g R A\right) \\
& =\chi_{Q}(\operatorname{det} D) \chi\left(\operatorname{det}{ }^{t} D\right) g^{\nu m} \sum_{R \in \mathbb{Z}_{m}^{m} / g \mathbb{Z}_{m}^{m}} \chi(\operatorname{det} R) \Theta_{\nu}\left(Z, g^{2} Q \mid q g R\right) \\
& =\left(\chi_{Q}\right)(M) \Theta_{\nu}(Z, Q, \chi) .
\end{aligned}
$$

The rest follows from Theorem 2 .

\section{§3. HECKE OpERATORS}

Here we recall the basic definitions concerning Hecke operators on the spaces $\mathfrak{M}_{k}^{n}(q)$ and specialize the formulas (obtained in [6]) for the transformation of general harmonic theta series under regular Hecke operators to the cases of the series $\Theta_{P}(Z, Q \mid L)$ and the Maass theta series. For the details and proofs, see Chapters 3 and 4 in the book [2] and [6, §5].

Let $\Delta$ be a multiplicative semigroup, and let $S$ be a subgroup of $\Delta$ such that every double coset $S M S$ of $\Delta$ modulo $S$ is a finite union of left cosets $S M^{\prime}$. We consider the vector space over a field (say, the field $\mathbb{C}$ of complex numbers) consisting of all formal finite linear combinations with coefficients in $\mathbb{C}$ of the symbols $(S M)$ with $M \in \Delta$, which are in one-to-one correspondence with the left cosets $S M$ of $\Delta$ modulo $S$. The group $S$ acts naturally on this space by right multiplication, defined on the symbols $(S M)$ by

$$
(S M) \gamma=(S M \gamma) \quad(M \in \Delta, \gamma \in S) .
$$

We denote by

$$
\mathcal{H}(S, \Delta)=H S_{\mathbb{C}}(S, \Delta)
$$

the subspace of all $S$-invariant elements. The product of elements of $\mathcal{H}(S, \Delta)$, defined by the formula

$$
\left(\sum_{\alpha} a_{\alpha}\left(S M_{\alpha}\right)\right)\left(\sum_{\beta} b_{\beta}\left(S N_{\beta}\right)\right)=\sum_{\alpha, \beta} a_{\alpha} b_{\beta}\left(S M_{\alpha} N_{\beta}\right),
$$


does not depend on the choice of representatives $M_{\alpha} \in S M_{\alpha}$ and $N_{\beta} \in S N_{\beta}$ and turns $\mathcal{H}(S, \Delta)$ into an associative algebra over $\mathbb{C}$ with the unity element $\left(S 1_{S}\right)$; this algebra is called the Hecke-Shimura ring or $H S$-ring of $\Delta$ relative to $S$ (over $\mathbb{C}$ ). The elements

$$
(M)=(M)_{S}=\sum_{M_{i} \in S \backslash S M S}\left(S M_{i}\right) \quad(M \in \Delta),
$$

which are in one-to-one correspondence with the double cosets of $\Delta$ modulo $S$, belong to $\mathcal{H}(S, \Delta)$ and form a basis of this ring over $\mathbb{C}$. For brevity, the symbols $(S M)$ and $(M)$ will be referred to as left and double classes (of $\Delta$ modulo $S$ ), respectively.

We consider the semigroup

$$
\Sigma^{n}=\mathbb{G}^{n} \cap \mathbb{Z}_{2 n}^{2 n}=\left\{M \in \mathbb{Z}_{2 n}^{2 n} \mid{ }^{t} M J_{n} M=\mu(M) J_{n}, \mu(M)>0\right\}
$$

and its subsemigroups

$$
\left\{\begin{aligned}
\Sigma_{q}^{n} & =\left\{M \in \Sigma^{n} \mid \operatorname{gcd}(\mu(M), q)=1\right\}, \\
\Sigma_{0}^{n}(q) & =\left\{M=\left(\begin{array}{cc}
A & B \\
C & D
\end{array}\right) \in \Sigma_{q}^{n} \mid C \equiv 0(\bmod q)\right\}, \\
\Sigma^{n}(q) & =\left\{M \in \Sigma_{0}^{n}(q) \mid M \equiv\left(\begin{array}{cc}
\mu(M) 1_{n} & 0 \\
0 & 1_{n}
\end{array}\right)(\bmod q)\right\},
\end{aligned}\right.
$$

with $q \in \mathbb{N}$. It is easily seen that these semigroups satisfy the relation

$$
\Sigma_{0}^{n}(q)=\Gamma_{0}^{n}(q) \Sigma^{n}(q)=\Sigma^{n}(q) \Gamma_{0}^{n}(q) .
$$

If $S$ is a subgroup of finite index in the modular group $\Gamma^{n}$, then the pair $S, \Sigma^{n}$ satisfies the conditions of the definition of $H S$-rings, and so does each pair $S, \Delta$ with a semigroup $\Delta$ satisfying $S \subset \Delta \subset \Sigma^{n}$. Thus, we can define the corresponding Hecke-Shimura rings $\mathcal{H}(S, \Delta)$. We shall say that a group $S$ satisfying $\Gamma^{n}(q) \subset S \subset \Gamma^{n}$ is q-symmetric if $S \Sigma^{n}(q)=\Sigma^{n}(q) S$. For such an $S$, the $H S$-ring

$$
\mathcal{H}_{q r}(S)=\mathcal{H}(S, R(S)) \quad \text { with } \quad R(S)=S \Sigma^{n}(q)=\Sigma^{n}(q) S
$$

is called the q-regular $H S$-ring of $S$. By Theorem 3.3.3 in [2], all $q$-regular $H S$-rings of given genus $n$ are isomorphic. In particular, by (3.3), the group $\Gamma_{0}^{n}(q)$ is $q$-symmetric, and the $q$-regular $H S$-rings of $\Gamma_{0}^{n}(q)$ and $\Gamma^{n}(q)$,

$$
\begin{aligned}
& \mathcal{H}_{0}^{n}(q)=\mathcal{H}_{q r}\left(\Gamma_{0}^{n}(q)\right)=\mathcal{H}\left(\Gamma_{0}^{n}(q), \Sigma_{0}^{n}(q)\right), \\
& \mathcal{H}^{n}(q)=\mathcal{H}_{q r}\left(\Gamma^{n}(q)\right)=\mathcal{H}\left(\Gamma^{n}(q), \Sigma^{n}(q)\right),
\end{aligned}
$$

are isomorphic. The corresponding isomorphism can be defined as follows. Let

$$
T^{\prime}=\sum_{\alpha} a_{\alpha}\left(\Gamma_{0}^{n}(q) M_{\alpha}\right) \in \mathcal{H}_{0}^{n}(q),
$$

where the left classes $\left(\Gamma_{0}^{n}(q) M_{\alpha}\right)$ with $a_{\alpha} \neq 0$ are pairwise distinct. By (3.3), there is no loss of generality in assuming that all the representatives $M_{\alpha}$ of the left cosets $\Gamma_{0}^{n}(q) M_{\alpha}$ belong to $\Sigma^{n}(q)$. Then, obviously, we have

$$
\eta: T^{\prime} \mapsto T=\sum_{\alpha} a_{\alpha}\left(\Gamma^{n}(q) M_{\alpha}\right) \in \mathcal{H}^{n}(q),
$$

and the map $\eta$ is a homomorphic embedding of the ring $\mathcal{H}_{0}^{n}(q)$ in $\mathcal{H}^{n}(q)$. In fact, $\eta$ is a ring isomorphism, with the inverse isomorphism $\xi: \mathcal{H}^{n}(q) \mapsto \mathcal{H}_{0}^{n}(q)$ determined by the condition

$$
\xi: \sum_{\beta} b_{\beta}\left(\Gamma^{n}(q) N_{\beta}\right) \mapsto \sum_{\beta} b_{\beta}\left(\Gamma_{0}^{n}(q) N_{\beta}\right) .
$$


The isomorphism of these rings makes it possible to transfer various constructions from one of the rings to another. For example, the Zharkovskaya homomorphisms $\Psi^{n, r}=\Psi_{k, \chi}^{n, r}$ of the ring $\mathcal{H}_{0}^{n}(q)$ to $\mathcal{H}_{0}^{r}(q)$, where $n>r \geq 1, k$ is an integer, and $\chi$ is a Dirichlet character modulo $q$ satisfying $\chi(-1)=(-1)^{k}$, can be carried over to the rings $\mathcal{H}^{n}(q)$, so that we get the homomorphisms

$$
\Psi^{n, r}=\Psi_{k, \chi}^{n, r}: \mathcal{H}^{n}(q) \mapsto \mathcal{H}^{r}(q) \quad(n>r \geq 1)
$$

making the diagram

$$
\begin{array}{lll}
\mathcal{H}_{0}^{n}(q) & \stackrel{\eta}{\longrightarrow} & \mathcal{H}^{n}(q) \\
\Psi^{n, r} \downarrow & & \downarrow \Psi^{n, r} \\
\mathcal{H}_{0}^{r}(q) & \stackrel{\eta}{\longrightarrow} & \mathcal{H}^{r}(q)
\end{array}
$$

commutative. We recall that the Zharkovskaya map from genus $n$ to genus $r$,

$$
\Psi^{n, r}=\Psi_{k, \chi}^{n, r}: \mathcal{H}_{0}^{n}(q) \mapsto \mathcal{H}_{0}^{r}(q),
$$

can be defined in the following way. Let $T^{\prime} \in \mathcal{H}_{0}^{n}(q)$ be an element of the form (3.5). We may assume that each representative $M_{\alpha} \in \Gamma_{0}^{n}(q) \backslash \Sigma_{0}^{n}(q)$ is chosen in the form

$$
M_{\alpha}=\left(\begin{array}{cc}
A_{\alpha} & B_{\alpha} \\
0_{n} & D_{\alpha}
\end{array}\right) \quad \text { with } \quad D_{\alpha}=\left(\begin{array}{cc}
D_{\alpha}^{\prime} & * \\
\mathbf{0} & D_{\alpha}^{\prime \prime}
\end{array}\right) \quad \text { and } \quad D_{\alpha}^{\prime} \in \mathbb{Z}_{r}^{r} .
$$

If $A_{\alpha}=\left(\begin{array}{c}A_{\alpha}^{\prime} * \\ * * *\end{array}\right)$ and $B_{\alpha}=\left(\begin{array}{c}B_{\alpha}^{\prime} * \\ * * *\end{array}\right)$ with $(r \times r)$-blocks $A_{\alpha}^{\prime}$ and $B_{\alpha}^{\prime}$, then

$$
M_{\alpha}^{\prime}=\left(\begin{array}{cc}
A_{\alpha}^{\prime} & B_{\alpha}^{\prime} \\
0_{r} & D_{\alpha}^{\prime}
\end{array}\right) \in \Sigma_{0}^{r}(q)
$$

and we put

$$
\Psi_{k, \chi}^{n, r}\left(T^{\prime}\right)=\sum_{\alpha} a_{\alpha}\left|\operatorname{det} D_{\alpha}^{\prime \prime}\right|^{-k} \chi^{-1}\left(\left|\operatorname{det} D_{\alpha}^{\prime \prime}\right|\right)\left(\Gamma_{0}^{r}(q) M_{\alpha}^{\prime}\right) .
$$

Then, since the diagram (3.9) is commutative, we can define the Zharkovskaya map (3.8) by

$$
\Psi^{n, r}(T)=\left(\eta \Psi^{\prime} \xi\right)(T) \in \mathcal{H}^{r}(q) \quad\left(T \in \mathcal{H}^{n}(q)\right),
$$

with the maps $\eta$ and $\xi$ defined by (3.6) and (3.7), where $\Psi^{\prime}$ is the map (3.10).

Hecke-Shimura rings act on modular forms via the linear representation given by Hecke operators. For instance, let $F$ belong to the space $\mathfrak{M}_{k}(S)=\mathfrak{M}_{k}(S, 1)$ of modular forms of integral weight $k$ and trivial character $\chi=1$ for a subgroup $S$ of finite index in the modular group $\Gamma^{n}$, and let

$$
T=\sum_{\alpha} a_{\alpha}\left(S M_{\alpha}\right) \in \mathcal{H}\left(S, \Sigma^{n}\right) .
$$

Then the definition of the modular forms and $H S$-rings and properties (2.2) and (2.3) of the Petersson operators (2.1) show that the function

$$
F\|T=F\|_{k} T=\left.\sum_{\alpha} a_{\alpha} F\right|_{k} M_{\alpha}
$$

does not depend on the choice of representatives $M_{\alpha} \in S M_{\alpha}$ and again belongs to the space $\mathfrak{M}_{k}(S)$. These operators are called Hecke operators (of weight $k$ for the group $S)$. The Hecke operators corresponding to elements of regular $H S$-rings are said to be regular. In accordance with (2.2) and the definition of multiplication in $H S$-rings, the map $T \mapsto \| T$ is a linear representation of the ring $\mathcal{H}(S)$ on the space $\mathfrak{M}_{k}(S)$. The subspace $\mathfrak{N}_{k}(S)$ of cusp forms is invariant under all Hecke operators.

The following theorem expresses the images of harmonic theta series under regular Hecke operators in the form of linear combinations of similar theta series with coefficients given explicitly in terms of certain trigonometric sums. 
Theorem 4. Let $Q$ be an even positive definite matrix of even order $m$ and level $q$, and let $P$ be a homogeneous polynomial on $\mathbb{C}_{n}^{m}$ of exponent $\nu$ and harmonic with respect to the quadratic form with matrix $Q$. Suppose that

$$
T=\sum_{\alpha} a_{\alpha}\left(\Gamma^{n}(q) M_{\alpha}\right)
$$

is an element of the Hecke-Shimura ring $\mathcal{H}^{n}(q)$ with $n \geq 1$ such that all representatives $M_{\alpha} \in \Sigma^{n}(q)$ have the same multiplier $\mu\left(M_{\alpha}\right)=\mu>0$. Also, assume that, in the case where $n<m$, the element $T$ belongs to the image of the ring $\mathcal{H}^{m}(q)$ under the Zharkovskaya map (3.8) from genus $m$ to genus $n$ with $k=m / 2$ and with the character $\chi=\chi_{Q}$ defined in Theorem 1 ,

$$
T=\Psi_{m / 2, \chi_{Q}}^{m, n}(\widetilde{T}) \quad \text { with } \quad \widetilde{T} \in \mathcal{H}^{m}(q) \quad(n<m) .
$$

Then the image of the theta series (1.1) under the Hecke operator $\|_{\nu+m / 2} T$ can be written in the form

$$
\begin{aligned}
& \Theta_{P}(Z, Q \mid L) \|_{\nu+m / 2} T \\
& \quad=\sum_{D \in \Delta(Q, \mu) / \Lambda} I\left(D, Q, \Psi^{n, m}(T)\right) \Theta_{P \mid \mu^{-1} D}\left(Z, \mu^{-1} Q[D] \mid \mu D^{-1} L\right),
\end{aligned}
$$

where

$$
\begin{gathered}
\Delta(Q, \mu)=\left\{D \in \mathbb{Z}_{m}^{m} \mid \operatorname{det} D=\mu^{m / 2}, \mu^{-1} Q[D] \in \mathbb{E}^{m}\right\}, \quad \Lambda=\Lambda^{m}=S L_{m}(\mathbb{Z}), \\
\Psi^{n, m}(T)=\Psi_{m / 2, \chi_{Q}}^{n, m}(T)= \begin{cases}\Psi_{m / 2, \chi_{Q}}^{n, m}(T) & \text { if } n>m, \\
T & \text { if } n=m, \\
\widetilde{T} \in\left(\Psi_{m / 2, \chi_{Q}}^{m, n}\right)^{-1}(T) & \text { if } n<m,\end{cases}
\end{gathered}
$$

the $I(D, Q, \widetilde{T})$ with $\widetilde{T} \in \mathcal{H}^{m}(q)$ are the trigonometric sums defined by the following conditions: if

$$
T^{\prime}=\xi(\widetilde{T})=\sum_{\beta} b_{\beta}\left(\Gamma_{0}^{m}(q)\left(\begin{array}{cc}
A_{\beta} & B_{\beta} \\
0 & D_{\beta}
\end{array}\right)\right) \in \mathcal{H}_{0}^{m}(q)
$$

with ${ }^{t} A_{\beta} D_{\beta}=\mu 1_{m}$, then

$$
\begin{aligned}
& I(D, Q, \widetilde{T})=I\left(D, Q, T^{\prime}\right) \\
& \quad=\sum_{\beta ; D^{t} D_{\beta} \equiv 0(\bmod \mu)} b_{\beta}\left|\operatorname{det} D_{\beta}\right|^{-m / 2} \chi_{Q}^{-1}\left(\left|\operatorname{det} D_{\beta}\right|\right) e\left\{\mu^{-2} Q[D]^{t} D_{\beta} B_{\beta}\right\}
\end{aligned}
$$

$(e\{\cdots\}$ is the exponent (1.3)), and where

$$
\left(P \mid \mu^{-1} D\right)(V)=P\left(\mu^{-1} D V\right) .
$$

In particular, the image of the theta series (1.5) with $n=m$ and of the theta series (1.6) under the Hecke operator $\|_{\nu+m / 2} T$ with $T \in \mathcal{H}^{m}(q)$ can be written in the form

$$
\Theta_{\nu}(Z, Q \mid L) \|_{\nu+m / 2} T=\mu^{-\nu m / 2} \sum_{D \in \Delta(Q, \mu) / \Lambda} I(D, Q, T) \Theta_{\nu}\left(Z, \mu^{-1} Q[D] \mid \mu D^{-1} L\right),
$$

where $\nu=0$ in the first case and $\nu=1$ in the second.

Proof. Formulas (3.11) were proved in [6, Theorem 5.3] with the summation taken over the sets of representatives

$$
\left\{D \in \mathbb{Z}_{m}^{m}|| \operatorname{det} D \mid=\mu^{m / 2}, \mu^{-1} Q[D] \in \mathbb{E}^{m}\right\} / G L_{m}(\mathbb{Z}) .
$$


Since, clearly, each set of this type can be replaced by the set $\{D \in \Delta(Q, \mu) / \Lambda\}$, the formulas follow.

As to formulas (3.14), it suffices to observe that $\operatorname{det}\left(\mu^{-1} D\right)=\mu^{-m / 2}$ for all $D \in$ $\Delta(Q, \mu)$.

In connection with formulas (3.11), we note that

$$
\mu D^{-1} \in \Delta\left(\mu^{-1} Q[D], \mu\right) \quad \text { if } \quad D \in \Delta(Q, \mu) \quad \text { and } \quad \operatorname{gcd}(\mu, q)=1 .
$$

Indeed, set $D^{\prime}=\mu D^{-1}$ and $Q^{\prime}=\mu^{-1} Q[D]$. Then the matrix $D^{\prime}$ can also be written as $\left\{Q^{\prime}\right\}^{-1} D Q$. It follows that the products of $D^{\prime}$ by $\operatorname{det} D=\mu^{m / 2}$ or $\operatorname{det} Q^{\prime}=\operatorname{det} Q$ are both integral. Since these numbers are coprime, $D^{\prime}$ is integral. The inclusion follows.

Proposition 5. Let $Q$ be an even positive definite matrix of even order $m, g$ a positive integer, and $\chi$ a Dirichlet character modulo $g$. Suppose that

$$
T=\sum_{\alpha} a_{\alpha}\left(\Gamma^{n}\left(q g^{2}\right) M_{\alpha}\right)
$$

is an element of the regular Hecke-Shimura ring $\mathcal{H}^{m}\left(q g^{2}\right)$ and that all representatives $M_{\alpha} \in \Sigma^{n}\left(q g^{2}\right)$ (see (3.2)) have the same multiplier $\mu\left(M_{\alpha}\right)=\mu$. Then the image of the Maass theta series $(*)$ under the Hecke operator $\|_{\nu+m / 2} T$ is again a linear combination of Maass theta series of the form

$$
\begin{aligned}
& \Theta_{\nu}(Z, Q, \chi) \|_{\nu+m / 2} T \\
& \quad=\chi(\mu)^{-m / 2} \mu^{-\nu m / 2} \sum_{D \in \Delta(Q, \mu) / \Lambda} I\left(D, g^{2} Q, T\right) \Theta_{\nu}\left(Z, \mu^{-1} Q[D], \chi\right),
\end{aligned}
$$

where the $I(\cdots)$ are the trigonometric sums (3.13).

Proof. By (1.7) and (3.14), we have

$$
\begin{aligned}
\Theta_{\nu}(Z, Q, \chi)\left\|_{\nu+m / 2} T=g^{\nu m} \sum_{R \in \mathbb{Z}_{m}^{m} / g \mathbb{Z}_{m}^{m}} \chi(\operatorname{det} R) \Theta_{\nu}\left(Z, g^{2} Q \mid q g R\right)\right\|_{\nu+m / 2} T \\
=\mu^{-\nu m / 2} g^{\nu m} \sum_{R \in \mathbb{Z}_{m}^{m} / g \mathbb{Z}_{m}^{m}} \chi(\operatorname{det} R) \\
\quad \times \sum_{D \in \Delta\left(g^{2} Q, \mu\right) / \Lambda} I\left(D, g^{2} Q, T\right) \Theta_{\nu}\left(Z, \mu^{-1} g^{2} Q[D] \mid q g \mu D^{-1} R\right) \\
=\mu^{-\nu m / 2} g^{\nu m} \sum_{D \in \Delta\left(g^{2} Q, \mu\right) / \Lambda} I\left(D, g^{2} Q, T\right) \\
\quad \times \sum_{R \in \mathbb{Z}_{m}^{m} / g \mathbb{Z}_{m}^{m}} \chi\left(\operatorname{det} \mu D^{-1} R\right) \chi\left(\operatorname{det} \mu D^{-1}\right)^{-1} \Theta_{\nu}\left(Z, \mu^{-1} g^{2} Q[D] \mid q g \mu D^{-1} R\right) \\
=\chi(\mu)^{-m / 2} \mu^{-\nu m / 2} \sum_{D \in \Delta(Q, \mu) / \Lambda} I\left(D, g^{2} Q, T\right) \\
\quad \times g^{\nu m} \sum_{R^{\prime} \in \mathbb{Z}_{m}^{m} / g \mathbb{Z}_{m}^{m}} \chi\left(\operatorname{det} R^{\prime}\right) \Theta_{\nu}\left(Z, \mu^{-1} g^{2} Q[D] \mid q g R^{\prime}\right) \\
=\chi(\mu)^{-m / 2} \mu^{-\nu m / 2} \sum_{D \in \Delta(Q, \mu) / \Lambda} I\left(D, g^{2} Q, T\right) \Theta_{\nu}\left(Z, \mu^{-1} Q[D], \chi\right),
\end{aligned}
$$

because, clearly, $\Delta\left(g^{2} Q, \mu\right)=\Delta(Q, \mu)$ if $\mu$ is prime to $q g^{2}$, the matrix $R^{\prime}=\mu D^{-1} R$ runs (together with $R$ ) through the set $\mathbb{Z}_{m}^{m} / g \mathbb{Z}_{m}^{m}$, and the level of $\mu^{-1} Q[D]$ with $D \in \Delta(Q, \mu)$ is again $q$. 
It is sometimes convenient to rewrite the above transformation formulas in a somewhat different form. For this, we need several preliminary remarks. We say that an even matrix $Q^{\prime}$ is properly similar to a nonsingular matrix $Q \in \mathbb{E}^{m}, Q^{\prime} \sim_{p} Q$, if $Q^{\prime}=\mu^{-1} Q[D]$ with $D \in \Delta(Q, \mu)$, where $\mu$ is coprime with the level $q$ of $Q$. From (3.15), it follows that the relation of proper similarity is symmetric. Moreover, it is clearly reflexive and transitive. Thus, the set of all nonsingular matrices of $\mathbb{E}^{m}$ is a disjoint union of ps-classes

$$
p s\{Q\}=\left\{Q^{\prime} \in \mathbb{E}^{m} \mid Q^{\prime} \sim_{p} Q\right\} \quad\left(Q \in \mathbb{E}^{m}, \operatorname{det} Q \neq 0\right) .
$$

It is easily seen that all matrices of the $p s$-class of a matrix $Q$ have the same signature, determinant, level, and divisor as $Q$. Next, we recall that two matrices $Q, Q^{\prime}$ in $\mathbb{E}^{m}$ are said to be properly equivalent, $Q \simeq_{p} Q^{\prime}$, if $Q^{\prime}=Q[U]$ with $U \in \Lambda=S L_{m}(\mathbb{Z})$. All matrices properly equivalent to a matrix $Q$ form the pe-class of $Q$,

$$
p e\{Q\}=\left\{Q^{\prime}=Q[U] \mid U \in \Lambda\right\} .
$$

In accordance with the reduction theory for integral quadratic forms, the $p s$-class of every nonsingular matrix $Q \in \mathbb{E}^{m}$ is a finite union of pe-classes:

$$
p s\{Q\}=\bigcup_{j=1}^{h_{p}(Q)} p e\left\{Q_{j}\right\} .
$$

The quantity $h_{p}(Q)$ will be called the proper class number of $Q$.

Proposition 6. With the notation and under the assumptions of Theorem 4, formula (3.11) can be rewritten as follows:

$$
\begin{aligned}
& \Theta_{P}(Z, Q \mid L) \|_{\nu+m / 2} T \\
& \quad=\sum_{j=1}^{h_{p}(Q)} \sum_{D \in R_{p}\left(Q, \mu Q_{j}\right) / E_{p}\left(Q_{j}\right)} I\left(D, Q, \Psi^{n, m}(T)\right) \Theta_{P \mid \mu^{-1} D}\left(Z, Q_{j} \mid \mu D^{-1} L\right) .
\end{aligned}
$$

Here $Q_{1}, \ldots, Q_{h_{p}(Q)}$ is a system of representatives of all different pe-classes contained in the ps-class of $Q$,

$$
R_{p}\left(Q, Q^{\prime}\right)=\left\{M \in \mathbb{Z}_{m}^{m} \mid Q[M]=Q^{\prime}, \operatorname{det} M>0\right\} \quad\left(Q, Q^{\prime} \in \mathbb{E}^{m}\right)
$$

is the set of all proper integral representations of $Q^{\prime}$ by $Q$, and

$$
E_{p}\left(Q^{\prime}\right)=R_{p}\left(Q^{\prime}, Q^{\prime}\right) \quad\left(Q^{\prime} \in \mathbb{E}^{m}, \operatorname{det} Q^{\prime} \neq 0\right)
$$

denotes the group of all proper units of $Q^{\prime}$.

In particular, formula (3.14) can be rewritten as

$$
\begin{aligned}
& \Theta_{\nu}(Z, Q \mid L) \|_{\nu+m / 2} T \\
& \quad=\mu^{-\nu m / 2} \sum_{j=1}^{h_{p}(Q)} \sum_{D \in R_{p}\left(Q, \mu Q_{j}\right) / E_{p}\left(Q_{j}\right)} I(D, Q, T) \Theta_{\nu}\left(Z, Q_{j} \mid \mu D^{-1} L\right) .
\end{aligned}
$$

Proof. It is an easy consequense of the definitions that the sums (3.13) are independent of the choice of representatives in the decomposition of $T^{\prime}$ and satisfy

$$
I\left(U D U^{\prime}, Q, T^{\prime}\right)=I\left(D, Q[U], T^{\prime}\right) \quad \text { for all } \quad U, U^{\prime} \in G L_{m}(\mathbb{Z})
$$

(see 6, Lemma 3.1]). Relations (1.4) and (3.20) show that the term of the sum on the right in (3.11) corresponding to a matrix $D \in \Delta(Q, \mu)$ depends only on the coset $D \Lambda$. On the other hand, each of the matrices $\mu^{-1} Q[D]$ with $D \in \Delta(Q, \mu)$ is properly similar to $Q$; so it is properly equivalent to one of the matrices $Q_{1}, \ldots, Q_{h_{p}(Q)}$, say, $\mu^{-1} Q[D] \simeq{ }_{p} Q_{j}$. This means that $\mu^{-1} Q[D][U]=\mu^{-1} Q[D U]=Q_{j}$ with $U \in \Lambda$. Replacing $D$ by $D U$, 
we may assume that $D \in R_{p}\left(Q, Q_{j}\right)$. If $D^{\prime}=D U^{\prime}$ is another matrix of this sort, then, clearly, $Q_{j}\left[U^{\prime}\right]=Q_{j}$, whence $U^{\prime} \in E_{p}\left(Q_{j}\right)$. This proves the formulas.

Similarly, in the same notation we have the following formulas for the action of regular Hecke operators on Maass theta series.

Proposition 7. In the notation and under the assumption of Proposition 5, formulas (3.16) can be rewritten as follows:

$$
\begin{aligned}
& \Theta_{\nu}(Z, Q, \chi) \|_{\nu+m / 2} T \\
& \quad=\chi(\mu)^{-m / 2} \mu^{-\nu m / 2} \sum_{j=1}^{h_{p}(Q)} \sum_{D \in R_{p}\left(Q, \mu Q_{j}\right) / E_{p}\left(Q_{j}\right)} I\left(D, g^{2} Q, T\right) \Theta_{\nu}\left(Z, Q_{j}, \chi\right) .
\end{aligned}
$$

Here $Q_{1}, \ldots, Q_{h_{p}(Q)}$ is a system of representatives of all different pe-classes contained in the ps-class of $Q$, and $R_{p}\left(Q, Q^{\prime}\right), E_{p}\left(Q^{\prime}\right)$ are the sets defined in Proposition 6.

In particular, if $h_{p}(Q)=1$, then, for each $\nu=0,1$ and every Dirichlet character $\chi$, the Maass series $\Theta_{\nu}(Z, Q, \chi)$ is an eigenfunction of the operator $\|_{\nu+m / 2} T$ with the eigenvalue

$$
\chi(\mu)^{-m / 2} \mu^{-\nu m / 2} \sum_{D \in R_{p}(Q, \mu Q) / E_{p}(Q)} I\left(D, g^{2} Q, T\right) .
$$

We conclude this section with a summary of the known results about computation of the sums (3.13). We recall that the $H S$-ring $\mathcal{H}_{0}^{n}(q)$ is generated over $\mathbb{C}$ by the following double classes of the form (3.1):

$$
T^{n}(p)=(\operatorname{diag}(\underbrace{1, \ldots, 1}_{n}, \underbrace{p, \ldots, p}_{n}))_{\Gamma_{0}^{n}(q)}
$$

and

$$
T_{j}^{n}\left(p^{2}\right)=\left(\operatorname{diag}(\underbrace{1, \ldots, 1}_{n-j}, \underbrace{p, \ldots, p}_{j}, \underbrace{p^{2}, \ldots, p^{2}}_{n-j}, \underbrace{p, \ldots, p}_{j})\right)_{\Gamma_{0}^{n}(q)} \quad(j=1, \ldots, n),
$$

where $p$ runs over all prime numbers not dividing $q$ (see [2, Theorem 3.3.23]). It follows that the ring $\mathcal{H}^{n}(q)$ is generated over $\mathbb{C}$ by the classes

$$
\widetilde{T}^{n}(p)=\eta\left(T^{n}(p)\right) \quad \text { and } \quad \widetilde{T}_{j}^{n}\left(p^{2}\right)=\eta\left(T_{j}^{n}\left(p^{2}\right)\right) \quad(j=1, \ldots, n),
$$

where $\eta$ is the map (3.6) and $p$ again runs over all prime numbers not dividing $q$.

Proposition 8. Let $Q$ be an even positive definite matrix of even order $m, q$ the level of $Q$, and $\chi_{Q}$ the Dirichlet character corresponding to $Q$. Then, for each prime number $p$ not dividing $q$, the sums (3.13) on the elements (3.22)-(3.24) can be computed by the following formulas:

$$
I\left(D, Q, \widetilde{T}^{m}(p)\right)=I\left(D, Q, T^{m}(p)\right)=p^{m / 2} \prod_{j=1}^{m / 2}\left(1+\chi_{Q}(p) p^{-j}\right)
$$


for all $D \in \Delta(Q, p)$;

$$
\begin{aligned}
& I\left(D, Q, \widetilde{T}_{m-1}^{m}(p)\right)=I\left(D, Q, T_{m-1}^{m}(p)\right) \\
& = \begin{cases}\chi_{Q}(p) p^{\left(2+m-m^{2}\right) / 2} \frac{\left(p^{m}-1\right)}{p-1}+p^{-m^{2} / 2}\left(\chi_{Q}(p) p^{m / 2}-1\right) & \text { if } D \in \Lambda\left(p 1_{m}\right), \\
\chi_{Q}(p) p^{\left(2+m-m^{2}\right) / 2} & \text { if } D \in \Lambda \operatorname{diag}(1, \underbrace{p, \ldots, p}_{m-2}, p^{2}) \Lambda, \\
0 & \text { otherwise } ;\end{cases} \\
& I\left(D, Q, \widetilde{T}_{m}^{m}\left(p^{2}\right)\right)=I\left(D, Q, T_{m}^{m}\left(p^{2}\right)\right)= \begin{cases}p^{-m^{2} / 2} & \text { if } D \in \Lambda\left(p 1_{m}\right), \\
0 & \text { otherwise, }\end{cases}
\end{aligned}
$$

where $\Lambda=S L_{m}(\mathbb{Z})$.

Proof. In 3] (formula (2.19) and Lemma 5.1 therein) certain sums $\gamma(Q, D, T)$ similar to the sums (3.11) were defined and computed for $T=T^{m}(p)$. In $\S 2$ of [4, the sums $\gamma(Q, D, T)$ were in fact computed for $T=T_{m}^{m}\left(p^{2}\right)=\left(p 1_{2 m}\right)_{\Gamma_{0}^{m}(q)}$ and $T=T_{m-1}^{m}\left(p^{2}\right)$ (see also [2, Lemma 3.3.32] for the presentation of $T_{m-1}^{m}\left(p^{2}\right)$ used in [4]). The definitions of the sums imply directly that $I(D, Q, T)=\mu^{m / 2} \gamma\left(Q, \mu D^{-1}, T\right)$. The rest is clear.

Note that the formulas of Proposition 7 determine the sums $I(D, Q, T)$ for all generators of the rings $\mathcal{H}_{0}^{2}(q)$ and $\mathcal{H}^{2}(q)$. We do not know of any other results on computation of the sums $I(D, Q, T)$.

\section{§4. THETA SERIES OF BINARY FORMS WITH CHARACTERS OF CLASS GROUPS}

In this section we shall construct eigenfunctions of regular Hecke operators in the form of linear combinations of Maass theta series of binary quadratic forms with characters of class groups of modules in the corresponding quadratic fields.

First, we recall the basic definitions and facts concerning modules in algebraic number fields and the relationship between quadratic modules and binary quadratic forms. For the details and proofs, see, e.g., [2, Appendix 3].

Let $\mathbf{K}$ be a (finite) algebraic number field, i.e., a finite extension of the field $\mathbb{Q}$. Any finitely generated $\mathbb{Z}$-submodule $\mathbf{M}$ of $\mathbf{K}$ is called a module in $\mathbf{K}$. Two modules $\mathbf{M}$ and $\mathbf{M}^{\prime}$ are said to be similar if $\mathbf{M}^{\prime}=\alpha \mathbf{M}$ for some element $\alpha \neq 0$ in $\mathbf{K}$. All modules in $\mathbf{K}$ split into classes of similar modules. A module $\mathbf{M}$ in $\mathbf{K}$ is said to be full if $\mathbb{Q} \mathbf{M}=\mathbf{K}$. Each full module in $\mathbf{K}$ has a $\mathbb{Z}$-basis of $n=[\mathbf{K}: \mathbb{Q}]$ elements.

A full module in $\mathbf{K}$ that contains 1 and is itself a ring is called an order in $\mathbf{K}$. Every order in $\mathbf{K}$ is contained in the maximal order $\mathcal{O}$ of all integers of $\mathbf{K}$ over $\mathbb{Q}$. If $\mathbf{M}$ is a module in $\mathbf{K}$, then the ring

$$
\mathcal{O}(\mathbf{M})=\{\alpha \in \mathbf{K} \mid \alpha \mathbf{M} \subset \mathbf{M}\}
$$

is called the ring of multipliers of $\mathbf{M}$. Similar modules have equal rings of multipliers. The ring of multipliers of a full module is an order in $\mathbf{K}$. For each full module, there is a similar module contained in its ring of multipliers.

Let $\mathcal{O}^{\prime}$ be an order in the field $\mathbf{K}$ and $\omega_{1}, \ldots, \omega_{n}$ a basis of $\mathcal{O}^{\prime}$. Then the number $d\left(\mathcal{O}^{\prime}\right)=\operatorname{det}\left(\operatorname{tr}\left(\omega_{i} \omega_{j}\right)\right)$, where tr means the trace from $\mathbf{K}$ to $\mathbb{Q}$, is independent of the choice of the basis and is called the discriminant of $\mathcal{O}^{\prime}$. The discriminant of the maximal order $\mathcal{O}$,

$$
d=d_{K}=d(\mathcal{O}),
$$

is called the (absolute) discriminant of $\mathbf{K}$. 
For a full module $\mathbf{M}$ with the ring of multipliers $\mathcal{O}^{\prime}=\mathcal{O}(\mathbf{M})$, let $\alpha_{1}, \ldots, \alpha_{n}$ and $\omega_{1}, \ldots, \omega_{n}$ be bases of $\mathbf{M}$ and $\mathcal{O}^{\prime}$, respectively. Then the absolute value of the determinant of the transition matrix from the first basis to the second,

$$
N(\mathbf{M})=\left|\operatorname{det}\left(a_{i j}\right)\right|, \quad \text { where } \alpha_{i}=\sum a_{i j} \omega_{j},
$$

is independent of the choice of bases, and is called the norm of $\mathbf{M}$. If $\mathbf{M} \subset \mathcal{O}^{\prime}$, then $N(\mathbf{M})=\left[\mathcal{O}^{\prime}: \mathbf{M}\right]$ (the index of $\mathbf{M}$ in $\mathcal{O}^{\prime}$ ).

Let $\mathbf{M}$ and $\mathbf{M}^{\prime}$ be two full modules in $\mathbf{K}$. Then the set $\mathbf{M} \mathbf{M}^{\prime}$ is again a full module in $\mathbf{K}$, which is called the product of $\mathbf{M}$ and $\mathbf{M}^{\prime}$. The norm of the product is equal to the product of the norms:

$$
N\left(\mathbf{M M}^{\prime}\right)=N(\mathbf{M}) N\left(\mathbf{M}^{\prime}\right) .
$$

All full modules in $\mathbf{K}$ with a fixed ring of multipliers $\mathcal{O}^{\prime}$ form a commutative group under multiplication of modules. The quotient of this group by the subgroup of modules similar to $\mathcal{O}^{\prime}$ is a finite group $H\left(\mathcal{O}^{\prime}\right)$ called the class group of $\mathcal{O}^{\prime}$.

Let $\mathcal{O}^{\prime}$ be an order in an algebraic number field. The full modules in $\mathbf{K}$ with the ring of multipliers $\mathcal{O}^{\prime}$ contained in $\mathcal{O}^{\prime}$ are called the regular ideals of $\mathcal{O}^{\prime}$. Each regular ideal of a given order in a finite extension $\mathbf{K}$ of $\mathbb{Q}$ is uniquely (up to order) decomposable in a product of regular prime ideals.

Now, let $\mathbf{K}$ be a quadratic extension of $\mathbb{Q}$. Every such field $\mathbf{K}$ is of the form $\mathbf{K}=$ $\mathbb{Q}\left(\sqrt{d_{0}}\right)$, where $d_{0} \neq 0$, and 1 is a square-free rational integer. As a basis of the maximal order $\mathcal{O}$ of $\mathbf{K}$, we can take the numbers 1 and $\omega$, where $\omega=\left(1+\sqrt{d_{0}}\right) / 2$ if $d_{0} \equiv 1$ $(\bmod 4)$, and $\omega=\sqrt{d_{0}}$ if $d_{0} \equiv 2$ or $3(\bmod 4)$. The discriminant $d=d_{K}=d(\mathbf{O})$ of the field $\mathbf{K}$ and the ring $\mathbf{O}$ is equal to $d_{0}$ in the first case and to $4 d_{0}$ in the second, and in both cases we can write $\mathbf{K}=\mathbb{Q}(\sqrt{d})$. Any order $\mathcal{O}^{\prime}$ of $\mathbf{K}$ has the form

$$
\mathcal{O}_{l}=\mathbb{Z}+l \omega \mathbb{Z},
$$

where $l$ is the index $\left[\mathcal{O}: \mathcal{O}^{\prime}\right]$. The discriminant of $\mathcal{O}_{l}$ is equal to $d l^{2}$.

For a full module $\mathbf{M}$ in $\mathbf{K}$, we denote by $\overline{\mathbf{M}}$ the module consisting of the conjugates $\bar{\alpha}$ over $\mathbb{Q}$ of the elements $\alpha \in \mathbf{M}$. The conjugate module $\overline{\mathbf{M}}$ is again a full module with the same ring of multipliers as $\mathbf{M}$, and we have

$$
\mathbf{M} \overline{\mathbf{M}}=N(\mathbf{M}) \mathcal{O}(\mathbf{M}) \text {. }
$$

Let $\mathbf{K}$ be a quadratic extension of $\mathbb{Q}$. For $\alpha, \beta, \ldots \in \mathbf{K}$, we shall denote by $\{\alpha, \beta, \ldots\}$ the module in $\mathbf{K}$ generated over $\mathbb{Z}$ by $\alpha, \beta, \ldots$ :

$$
\{\alpha, \beta, \ldots\}=\mathbb{Z} \alpha+\mathbb{Z} \beta+\cdots .
$$

The following lemma, which was proved, e.g., in [2, Lemma A.3.2], is often useful for the study of quadratic modules.

Lemma 9. Let $\mathbf{K}$ be a quadratic field, let $\gamma \in \mathbf{K}, \gamma \notin \mathbb{Q}$, and let $a \gamma^{2}+b \gamma+c=0$, where $a, b$, and $c$ are rational integers satisfying $\operatorname{gcd}(a, b, c)=1$ and $a>0$. Then the module $\mathbf{M}=\{1, \gamma\}$ satisfies

$$
N(\mathbf{M})=1 / a, \quad \mathcal{O}(\mathbf{M})=\{1, a \gamma\}, \quad d(\mathcal{O}(\mathbf{M}))=b^{2}-4 a c .
$$

The relationship between quadratic modules and prime numbers is described in the following proposition (the proof can be found, e.g., in [1, Proposition 2.3.1] or [2, Proposition A.3.5]).

Proposition 10. Let $\mathbf{K}=\mathbb{Q}(\sqrt{d})$ be the quadratic field with discriminant d, and let $\mathcal{O}_{l}$ be the order of $\mathbf{K}$ with discriminant $d^{2}$. Suppose that $p$ is a rational prime number not dividing $\mathbf{l}$. Then the existence of a full module $\mathbf{M}$ in $\mathbf{K}$ satisfying

$$
\mathcal{O}(\mathbf{M})=\mathcal{O}_{l}, \quad \mathbf{M} \subset \mathcal{O}_{l}, \quad N(\mathbf{M})=p
$$


is equivalent to the solvability of the congruence $x^{2} \equiv d(\bmod 4 p)$. If this congruence is solvable and $p$ does not divide $d$, then there are precisely two modules $\mathfrak{p}$ and $\mathfrak{p}^{\prime}$ with properties (4.3), and $\mathfrak{p}^{\prime}=\overline{\mathfrak{p}}$. But if the congruence is solvable and $p$ divides $d$, then there is exactly one module $\mathfrak{p}$ satisfying (4.3), and $\overline{\mathfrak{p}}=\mathfrak{p}$. Finally, if the congruence has no solutions, then there is a unique complete module $\mathfrak{p}$ satisfying

$$
\mathcal{O}(\mathfrak{p})=\mathcal{O}_{l}, \quad \mathfrak{p} \subset \mathcal{O}_{l}, \quad N(\mathfrak{p})=p^{2},
$$

and $\mathfrak{p}=p \mathcal{O}_{l}$. All the modules $\mathbf{M}=\mathfrak{p}$ listed above are regular prime ideals of the ring $\mathcal{O}_{l}$.

We conclude the survey part of this section with a description of the correspondence between modules in quadratic fields and integral binary quadratic forms. We restrict ourselves to imaginary quadratic fields and positive definite forms, because for applications we shall not need any others.

Let

$$
\mathbf{q}(X)=\frac{1}{2} Q[X]=a x^{2}+b x y+c y^{2} \quad\left(X=\left(\begin{array}{l}
x \\
y
\end{array}\right)\right)
$$

be a binary quadratic form with matrix $Q=\left(\begin{array}{cc}2 a & b \\ b & 2 c\end{array}\right)$. The number $d(\mathbf{q})=d(Q)=b^{2}-4 a c$ is called the discriminant of $\mathbf{q}$ and $Q$. The form is said to be integral if $Q \in \mathbb{E}^{2}$, i.e., if $a$, $b$, and $c$ are rational integers. In this case $\operatorname{gcd}(a, b, c)$ is the divisor of $\mathbf{q}$, and the form is primitive if the divisor equals 1 . We recall that two forms $\mathbf{q}$ and $\mathbf{q}^{\prime}$ with matrices $Q$ and $Q^{\prime}$ are properly equivalent if

$$
\mathbf{q}(U X)=\mathbf{q}^{\prime}(X) \quad \text { or } \quad{ }^{t} U Q U=Q^{\prime} \quad \text { with } U \in \Lambda^{2}=S L_{2}(\mathbb{Z}) .
$$

In accordance with the reduction theory of integral quadratic forms (see, e.g., [2, Theorem 2.1.12]), the set of all integral binary forms with a fixed nonzero discriminant $d$ is a finite union of classes (3.17) of properly equivalent forms. All forms of a fixed class have the same signature, level, discriminant, and divisor.

Let $\mathbf{K}=\mathbb{Q}(\sqrt{d})$ be an imaginary quadratic field with discriminant $d<0$, and let $\mathbf{M}$ be a full module in $\mathbf{K}$ with the ring of multipliers $\mathcal{O}(\mathbf{M})=\mathcal{O}_{l}$. With every $\mathbb{Z}$-basis $\alpha, \beta$ of $\mathbf{M}$ ordered by the condition $\Im(\beta / \alpha)>0$, we associate the binary quadratic form

$$
\mathbf{q}=q(\mathbf{M})(X)=\frac{1}{N(M)}(\alpha x+\beta y)(\bar{\alpha} x+\bar{\beta} y)=a x^{2}+b x y+c y^{2} .
$$

Clearly, the form $\mathbf{q}$ is positive definite. From Lemma 9 it follows that $\mathbf{q}$ is integral and primitive, with the discriminant $d(\mathbf{q})=b^{2}-4 a c=d l^{2}$ equal to the discriminant of the ring of multipliers $\mathcal{O}(\mathbf{M})$. Conversely, if $\mathbf{q}(X)=a x^{2}+b x y+c y^{2}$ is a positive definite integral primitive quadratic form with discriminant $b^{2}-4 a c=d l^{2}$, then the module

$$
\mathbf{M}=M(\mathbf{q})=\left\{a, \frac{-b+l \sqrt{d}}{2}\right\}
$$

is a full module in $\mathbf{K}$ with the ring of multipliers $\mathcal{O}_{l}$ contained in this ring. It is not difficult to check that the correspondences indicated above determine a bijection between the set of all classes of similar full modules in $\mathbf{K}$ with the ring of multipliers $\mathcal{O}_{l}$ and the set of all classes of properly equivalent positive definite integral primitive binary quadratic forms of discriminant $d l^{2}$.

Let $\mathbf{q}$ and $\mathbf{q}^{\prime}$ be two integral primitive positive definite binary quadratic forms of the same negative discriminant $-\delta$, and let $Q=Q(\mathbf{q})$ and $Q^{\prime}=Q\left(\mathbf{q}^{\prime}\right)$ be the matrices of these forms. Since the modules $\mathbf{M}=M(\mathbf{q})$ and $\mathbf{M}^{\prime}=M\left(\mathbf{q}^{\prime}\right)$ are full modules in the field $\mathbf{K}=\mathbb{Q}(\sqrt{-\delta})$ with the same ring of multipliers $\mathcal{O}_{l}$ of discriminant $-\delta=d l^{2}$, where $d$ is 
the discriminant of the field $\mathbf{K}$, the same is true for their product $\mathbf{M} \mathbf{M}^{\prime}$. We define the product $\mathbf{q} \times \mathbf{q}^{\prime}$ of two forms $\mathbf{q}$ and $\mathbf{q}^{\prime}$ by

$$
\mathbf{q} \times \mathbf{q}^{\prime}=q\left(\mathbf{M} \mathbf{M}^{\prime}\right)
$$

and denote by $Q \times Q^{\prime}$ the matrix of $\mathbf{q} \times \mathbf{q}^{\prime}$ :

$$
Q \times Q^{\prime}=Q\left(\mathbf{q} \times \mathbf{q}^{\prime}\right) .
$$

Next, for any full module $\mathbf{N}$ in $\mathbf{K}$ with the ring of multipliers $\mathcal{O}_{l}$, we set

$$
\mathbf{q} \times \mathbf{N}=\mathbf{N} \times \mathbf{q}=q(M(\mathbf{q}) \mathbf{N})
$$

and denote by $Q \times \mathbf{N}$ the matrix of $\mathbf{q} \times \mathbf{N}$ :

$$
Q \times \mathbf{N}=\mathbf{N} \times Q=Q(\mathbf{q} \times \mathbf{N}) .
$$

The multiplication (4.5) is called the Gauss composition of quadratic forms. The proper equivalence class of the product $\mathbf{q} \times \mathbf{q}^{\prime}$ depends only on the proper classes of $\mathbf{q}$ and $\mathbf{q}^{\prime}$, and the proper equivalence class of $\mathbf{q} \times \mathbf{N}$ depends only on the proper class of $\mathbf{q}$ and the similarity class of $\mathbf{N}$. Thus, formulas (4.5)-(4.8) define the products of the corresponding classes.

Since the similarity classes of full modules with the ring of multipliers of discriminant $-\delta$ form an Abelian group under multiplication of classes, the set of proper equivalence classes of integral primitive positive definite binary quadratic forms with discriminant $-\delta$ can also be endowed with a group structure. We denote by $H(-\delta)$ these two class groups.

The following useful lemma is an easy consequence of the definitions.

Lemma 11. The level $q$ and the discriminant $-\delta$ of an integral primitive positive definite binary quadratic form with matrix $Q$ satisfy the relations $q=\operatorname{det} Q=\delta$.

Now we turn to the action of regular Hecke operators on Maass theta series (*) of genus 2 .

Theorem 12. Let $q$ be a positive integer, and let $Q_{1}, \ldots, Q_{h(-q)}$ be a system of representatives of all different proper classes (3.17) of even primitive positive definite matrices of order 2 and level $q$. Finally, let $\psi$ be a character of the group $H(-q)$ of proper equivalence classes of matrices for integral primitive positive definite binary quadratic forms of discriminant $-q$ under the composition (4.6), and let $\chi$ be a Dirichlet character modulo a rational integer $\mathrm{g}$. Then any linear combination of Maass theta series of the form

$$
F=\Theta_{\nu}(Z, q, \psi, \chi)=\sum_{j=1}^{h(-q)} \psi\left(Q_{j}\right) \Theta_{\nu}\left(Z, Q_{j}, \chi\right) \quad(\nu=0,1)
$$

has the following properties.

1) The function $F$ is independent of the choice of representatives $Q_{j}$ in the proper equivalence classes; it is a modular form of weight $\nu+1$ for the group $\Gamma_{0}^{2}\left(q g^{2}\right)$ with the character $\chi_{Q}$, where $\chi_{Q}$ is the character of an even primitive positive definite matrix $Q$ of order 2 and level $q$, defined in Theorem 1 ; if $\nu=1$, then $F$ is a cusp form.

2) The modular form $F$ is an eigenfunction for all Hecke operators of the regular Hecke-Shimura ring $\mathcal{H}^{2}\left(q g^{2}\right)$ :

$$
F \|_{\nu+1} T=\lambda_{F}(T) F \quad\left(\forall T \in \mathcal{H}^{2}\left(q g^{2}\right)\right) .
$$


3) For each prime number $p$ not dividing qg, the eigenvalues of the operators corresponding to the generators (3.24) of the ring $\mathcal{H}^{2}\left(q g^{2}\right)$ can be given by the formulas

$$
\begin{gathered}
\lambda_{F}\left(\widetilde{T}^{2}(p)\right)= \begin{cases}\frac{(p+1)(\psi(\mathfrak{p})+\psi(\overline{\mathfrak{p}}))}{\chi(p) p^{\nu}} & \text { if } \chi_{Q}(p)=1, \\
0 & \text { if } \chi_{Q}(p)=-1 ;\end{cases} \\
\lambda_{F}\left(\widetilde{T}_{1}^{2}\left(p^{2}\right)\right)= \begin{cases}\frac{\left(p^{3}+p^{2}+p-1+(p \psi(\mathfrak{p}))^{2}+(p \psi(\overline{\mathfrak{p}}))^{2}\right)}{\chi(p)^{2} p^{2(\nu+1)}} & \text { if } \chi_{Q}(p)=1, \\
-\frac{\left(p^{3}+p^{2}+p+1\right)}{\chi(p)^{2} p^{2(\nu+1)}} & \text { if } \chi_{Q}(p)=-1,\end{cases}
\end{gathered}
$$

where, in the case of $\chi_{Q}(p)=1$, in both formulas, $\mathfrak{p}$ and $\overline{\mathfrak{p}}$ are conjugate regular prime ideals of the order $\mathcal{O}^{\prime}$ with discriminant $-q$ in the field $\mathbf{K}=\mathbb{Q}(\sqrt{-q})$ that satisfy $\mathfrak{p \mathfrak { p }}=\mathcal{O}^{\prime}$, and

$$
\lambda_{F}\left(\widetilde{T}_{2}^{2}\left(p^{2}\right)\right)=\frac{1}{\chi(p)^{2} p^{2(\nu+1)}} .
$$

We start with some preliminary results.

Lemma 13. Each of the Maass series $(*)$ satisfies the relations

$$
\Theta_{\nu}(Z, Q[U], \chi)=\chi(\operatorname{det} U)^{-1}(\operatorname{det} U)^{-\nu} \Theta_{\nu}(Z, Q, \chi) \quad\left(\forall U \in G L_{m}(\mathbb{Z})\right) .
$$

In particular, such a series depends only on the proper equivalence class (3.17) of $Q$.

Proof. This follows directly from the definition, by the change $N \mapsto U^{-1} N$.

Lemma 14. Let $Q$ be an even primitive positive definite matrix of order $2, q$ the level of $Q$, and $\chi_{Q}$ the corresponding Dirichlet character modulo $q$. Let $d$ be the discriminant of the imaginary quadratic field $\mathbf{K}=\mathbb{Q}(\sqrt{-q})$, so that $-q=d l^{2}$, and let $\mathcal{O}_{l}$ be the order in $\mathbf{K}$ with discriminant $-q$. For a prime number $p$ not dividing $q$ and a positive integer $\beta$, let $\Pi\left(p^{\beta}\right)$ denote the linear operator given on the functions $\Phi$ of proper equivalence classes of matrices $Q^{\prime}$ properly similar to $Q$ by

$$
\begin{aligned}
& \left(\Phi \circ \Pi\left(p^{\beta}\right)\right)(Q)=\sum_{D \in\left\{\Lambda\left(\begin{array}{cc}
1 & 0 \\
0 & p^{\beta}
\end{array}\right) \Lambda \cap \Delta\left(Q, p^{\beta}\right)\right\} / \Lambda} \Phi\left(p^{-\beta} Q[D]\right), \\
& \left(\Phi \circ \Pi\left(p^{\beta}\right)\right)(Q)=0 \quad \text { if } \Lambda\left(\begin{array}{cc}
1 & 0 \\
0 & p^{\beta}
\end{array}\right) \Lambda \cap \Delta\left(Q, p^{\beta}\right)=\varnothing,
\end{aligned}
$$

where the $\Delta(Q, \mu)$ are the sets (3.12), and $\Lambda=\Lambda^{2}=S L_{2}(\mathbb{Z})$. Then:

1) If $\chi_{Q}(p)=1$, then there are precisely two regular prime ideals $\mathfrak{p}$ and $\mathfrak{p}^{\prime}$ of norm $p$ in the ring $\mathcal{O}_{l}$ conjugate to each other, $\mathfrak{p}^{\prime}=\overline{\mathfrak{p}}$, and

$$
\left(\Phi \circ \Pi\left(p^{\beta}\right)\right)(Q)=\Phi\left(Q \times \mathfrak{p}^{\beta}\right)+\Phi\left(Q \times \overline{\mathfrak{p}}^{\beta}\right),
$$

where $\times$ means the multiplication (4.8).

2) If $\chi_{Q}(p)=-1$, then the ring $\mathcal{O}_{l}$ contains no regular prime ideals of norm $p$, and

$$
\left(\Phi \circ \Pi\left(p^{\beta}\right)\right)(Q)=0 .
$$

Proof. First, on the set of all pairs $(u, v)$ of coprime integers we introduce an equivalence relation $\sim\left(\bmod p^{\beta}\right)$ as on the "projective line modulo $p^{\beta "}$ :

$$
\left(u^{\prime}, v^{\prime}\right) \sim(u, v) \quad\left(\bmod p^{\beta}\right) \Longleftrightarrow u^{\prime} \equiv t u, \quad v^{\prime} \equiv t v \quad\left(\bmod p^{\beta}\right),
$$

and denote by $R\left(p^{\beta}\right)$ a set of integral matrices $U$ of order 2 in which the first column runs through a system of representatives for the equivalence classes $\bmod p^{\beta}$ of all coprime 
pairs and the second column is fixed by the condition $U \in \Lambda$. Then it is easy to show that the following decomposition into different right cosets modulo $\Lambda$ is possible:

$$
\Lambda\left(\begin{array}{cc}
1 & 0 \\
0 & p^{\beta}
\end{array}\right) \Lambda=\bigcup_{U \in R\left(p^{\beta}\right)} U\left(\begin{array}{cc}
1 & 0 \\
0 & p^{\beta}
\end{array}\right) \Lambda .
$$

Now, let $\mathbf{q}(x, y)=a x^{2}+b x y+c y^{2}$ be the quadratic form with matrix $Q$. We consider the congruence

$$
\mathbf{q}(x, y)=a x^{2}+b x y+c y^{2} \equiv 0 \quad\left(\bmod p^{\beta}\right) .
$$

We say that a solution $(u, v)$ of this congruence is primitive if the integers $u, v$ are coprime. An easy consideration of the corresponding quadratic space over the field of $p$ elements shows that if $\chi_{Q}(p)=1$, then the congruence $x^{2} \equiv d(\bmod 4 p)$ is solvable, the congruence (4.14) has precisely two primitive solutions nonequivalent modulo $p^{\beta}$, and these solutions are already nonequivalent modulo $p$, but if $\chi_{Q}(p)=-1$, then the congruence $x^{2} \equiv d(\bmod 4 p)$ is unsolvable, and the congruence $(4.14)$ has no primitive solutions (see, e.g., [2, Proof of Proposition 4.3.14]).

On the other hand, note that a matrix of the form

$$
U\left(\begin{array}{cc}
1 & 0 \\
0 & p^{\beta}
\end{array}\right) \quad \text { with } \quad U=\left(\begin{array}{ll}
u & u_{1} \\
v & v_{1}
\end{array}\right) \in \Lambda
$$

belongs to the set $\Delta\left(Q, p^{\beta}\right)$ if and only if the pair $u, v$ is a primitive solution of (4.14). Hence, part 2) follows from Proposition 10.

Let $\chi_{Q}(p)=1$, and let $(u, v),\left(u^{\prime}, v^{\prime}\right)$ be two nonequivalent primitive solutions of (4.14). Since $p$ does not divide $q=\operatorname{det} Q$, we may assume that $\mathbf{q}(u, v)=p^{\beta} a_{1}$ and $\mathbf{q}\left(u^{\prime}, v^{\prime}\right)=p^{\beta} a_{2}$, where $a_{1}$ and $a_{2}$ are not divisible by $p$. We can choose the set $R\left(p^{\beta}\right)$ so that

For $i=1,2$, we set

$$
U_{1}=\left(\begin{array}{ll}
u & u_{1} \\
v & v_{1}
\end{array}\right) \in R\left(p^{\beta}\right) \quad \text { and } \quad U_{2}=\left(\begin{array}{cc}
u^{\prime} & u_{1}^{\prime} \\
v^{\prime} & v_{1}^{\prime}
\end{array}\right) \in R\left(p^{\beta}\right) .
$$

$$
Q_{i}=Q\left[U_{i}\right]=\left(\begin{array}{cc}
2 p^{\beta} a_{i} & b_{i} \\
b_{i} & 2 c_{i}
\end{array}\right), \quad Q_{i}^{\prime}=p^{-\beta} Q\left[U_{i}\left(\begin{array}{cc}
1 & 0 \\
0 & p^{\beta}
\end{array}\right)\right]=\left(\begin{array}{cc}
2 a_{i} & b_{i} \\
b_{i} & 2 p^{\beta} c_{i}
\end{array}\right) .
$$

Then, in accordance with (4.22), (4.25), and the above considerations, we can write

$$
\Phi(Q) \circ \Pi\left(p^{\beta}\right)=\Phi\left(Q_{1}^{\prime}\right)+\Phi\left(Q_{2}^{\prime}\right) .
$$

Now, consider the modules in $\mathbf{K}$ given by

$$
\mathbf{M}_{1}=\left\{p^{\beta}, \frac{-b_{1}+l \sqrt{d}}{2}\right\}, \quad \mathbf{M}_{2}=\left\{p^{\beta}, \frac{-b_{2}+l \sqrt{d}}{2}\right\}
$$

and show first that these modules are regular ideals of $\mathcal{O}_{l}$ of norm $p^{\beta}$. For example, consider the module $\mathbf{M}_{1}$. The number $\gamma_{1}=\left(-b_{1}+l \sqrt{d}\right) /\left(2 p^{\beta}\right)$ is a root of the polynomial $p^{\beta} x^{2}+b_{1} x+a_{1} c_{1}$. Since $q=4 a c-b^{2}=4 p^{\beta} a_{1} c_{1}-b_{1}^{2}$ is not divisible by $p$, it follows that $b_{1}$ is not divisible by $p$, and so the coefficients of the polynomial are coprime. Then, by Lemma 9, the ring of multipliers of the module $\mathbf{M}_{1}=p^{\beta}\left\{1, \gamma_{1}\right\}$ is $\mathcal{O}_{l}$, and for its norm we have $N\left(\mathbf{M}_{1}\right)=N\left(p^{\beta}\right) / p^{\beta}=p^{\beta}$. It is also clear that $\mathbf{M}_{1} \subset \mathcal{O}_{l}$. Next, we show that the ideals $\mathbf{M}_{1}$ and $\mathbf{M}_{2}$ are coprime, i.e., $\mathbf{M}_{1}+\mathbf{M}_{2}=\mathcal{O}_{l}$. Obviously, the numbers $\left(b_{2}-b_{1}\right) / 2$ and $p^{\beta}$ are both contained in $\mathbf{M}_{1}+\mathbf{M}_{2}$. Hence, every integral linear combination of these numbers is contained in the sum; in particular, $s=\operatorname{gcd}\left(\left(b_{2}-b_{1}\right) / 2, p^{\beta}\right)$ is contained in $\mathbf{M}_{1}+\mathbf{M}_{2}$. Thus, we only need to prove that $s=1$. We set

$$
U_{1}^{-1} U_{2}=\left(\begin{array}{cc}
v_{1} & -u_{1} \\
-v & u
\end{array}\right)\left(\begin{array}{cc}
u^{\prime} & u_{1}^{\prime} \\
v^{\prime} & v_{1}^{\prime}
\end{array}\right)=\left(\begin{array}{cc}
t_{1} & t_{2} \\
t_{3} & t_{4}
\end{array}\right)=T \in \Lambda .
$$


Since the pairs $(u, v)$ and $\left(u^{\prime}, v^{\prime}\right)$ are not equivalent modulo $p$, it follows that $t_{3}=$ $u v^{\prime}-v u^{\prime}$ is not divisible by $p$. By comparing the corresponding entries of the matrices in the obvious relation $Q_{1}[T]=Q_{1}\left[U_{1}^{-1} U_{2}\right]=Q_{2}$, we see that

$$
p^{\beta} a_{1} t_{1}^{2}+b_{1} t_{1} t_{3}+c_{1} t_{3}^{2}=p^{\beta} a_{2} \quad \text { and } \quad 2 p^{\beta} a_{1} t_{1} t_{2}+b_{1}\left(t_{1} t_{4}+t_{2} t_{3}\right)+2 c_{1} t_{3} t_{4}=b_{2} .
$$

Since $t_{3}$ is not divisible by $p$, the first relation implies that $b_{1} t_{1}+c_{1} t_{3} \equiv 0(\bmod p)$. Since $t_{1} t_{4}-t_{2} t_{3}=1$, the second relation implies that $\left(b_{2}-b_{1}\right) / 2 \equiv t_{3}\left(b_{1} t_{2}+c_{1} t_{4}\right)\left(\bmod p^{\beta}\right)$. Thus, if $\left(b_{2}-b_{1}\right) / 2$ is divisible by $p$, then we have the congruences

$$
b_{1} t_{1}+c_{1} t_{3} \equiv b_{1} t_{2}+c_{1} t_{4} \equiv 0 \quad(\bmod p)
$$

which imply the congruences $b_{1} \equiv c_{1} \equiv 0(\bmod p)$, contrary to the assumption that $q=$ $4 p^{\beta} a_{1} c_{1}-b_{1}^{2}$ is not divisible by $p$. By the theorem on prime ideal factorization of regular ideals of the $\operatorname{ring} \mathcal{O}_{l}$ and Proposition 10, what we have proved implies that $\mathbf{M}_{1}=\mathfrak{p}^{\beta}$ and $\mathbf{M}_{2}=\overline{\mathfrak{p}}^{\beta}$, where $\mathfrak{p}$ and $\overline{\mathfrak{p}}$ are the only regular prime ideals of norm $p$ in $\mathcal{O}_{l}$. Let $\mathbf{q}_{1}, \mathbf{q}_{2}$, $\mathbf{q}_{1}^{\prime}$, and $\mathbf{q}_{2}^{\prime}$ be the quadratic forms with the matrices $Q_{1}, Q_{2}, Q_{1}^{\prime}$, and $Q_{2}^{\prime}$, respectively, and let $M\left(\mathbf{q}_{1}\right), M\left(\mathbf{q}_{2}\right), M\left(\mathbf{q}_{1}^{\prime}\right)$, and $M\left(\mathbf{q}_{2}^{\prime}\right)$ be the modules (4.4) corresponding to these forms. We set $\delta_{1}=\left(-b_{1}+l \sqrt{d}\right) / 2$. Since, clearly, $\delta_{1}^{2}=-b_{1} \delta_{1}-p^{\beta} a_{1} c_{1}$ and, as was noted above, $b_{1}$ is prime to $p$, it follows that

$$
\begin{aligned}
M\left(\mathbf{q}_{1}^{\prime}\right) \mathfrak{p}^{\beta} & =\left\{a_{1}, \delta_{1}\right\}\left\{p^{\beta}, \delta_{1}\right\}=\left\{a_{1} p^{\beta}, a_{1} \delta_{1}, p^{\beta} \delta_{1},-b_{1} \delta_{1}-p^{\beta} a_{1} c_{1}\right\} \\
& =\left\{a_{1} p^{\beta}, \delta_{1}\right\}=M\left(\mathbf{q}_{1}\right) .
\end{aligned}
$$

Recalling (4.2), we obtain

$$
M\left(\mathbf{q}_{1}^{\prime}\right)=\frac{1}{p^{\beta}} M\left(\mathbf{q}_{1}^{\prime}\right) \mathfrak{p}^{\beta} \overline{\mathfrak{p}}^{\beta}=\frac{1}{p^{\beta}} M\left(\mathbf{q}_{1}\right) \overline{\mathfrak{p}}^{\beta} .
$$

Since the form $\mathbf{q}_{1}$ is properly equivalent to $\mathbf{q}$, the last module is similar to $M(\mathbf{q}) \overline{\mathfrak{p}}^{\beta}$. Thus, the matrix $Q_{1}^{\prime}$ of $\mathbf{q}_{1}^{\prime}$ is properly equivalent to the matrix $Q \times \overline{\mathfrak{p}}^{\beta}$. Similarly, $Q_{2}^{\prime}$ is properly equivalent to $Q \times \mathfrak{p}^{\beta}$.

Proof of Theorem 12. Part 1) follows from the definition of the function $F$ and Proposition 3 .

Since the elements (3.24) generate the ring $\mathcal{H}^{2}\left(q g^{2}\right)$ over $\mathbb{C}$, for the proof of parts 2) and 3 ) it suffices to show that the function $F$ is an eigenfunction for all Hecke operators corresponding to the elements $T$ of the form (3.24), with eigenvalues given by formulas (4.9)-(4.11).

By Proposition 5, we can write

$$
F \|_{\nu+1} T=\frac{1}{\chi(\mu) \mu^{\nu}} \sum_{j=1}^{h(-q)} \psi\left(Q_{j}\right) \sum_{D \in \Delta\left(Q_{j}, \mu\right) / \Lambda} I\left(D, g^{2} Q_{j}, T\right) \Theta_{\nu}\left(Z, \mu^{-1} Q_{j}[D], \chi\right),
$$

where $\mu=\mu(T)$ is equal to $p$ for the first of the elements (3.24) with $n=2$ and to $p^{2}$ for the second and the third element.

Let $T=\eta\left(T^{2}(p)\right)$. By the definition (3.13) of the sums $I(D, Q, T)$ and Proposition 8, for all $D \in \Delta\left(Q_{j}, p\right)$ we have

$$
I\left(D, Q_{j}, \eta\left(T^{2}(p)\right)\right)=I\left(D, Q_{j}, T^{2}(p)\right)=p\left(1+\chi_{Q}(p) p^{-1}\right)=p+\chi_{Q}(p),
$$

because all matrices $Q_{j}$ have the same character as the matrix $Q$ of part 1$)$. If the set $\Delta\left(Q_{j}, p\right)$ is not empty, then, clearly, it is contained in the double coset $\Lambda\left(\begin{array}{lll}1 & 0 \\ 0 & p\end{array}\right) \Lambda$. 
Therefore, using the notation (4.12), we can rewrite the above formula for $T=\eta\left(T^{2}(p)\right)$ in the form

$$
F \|_{\nu+1} T=\frac{p+\chi_{Q}(p)}{\chi(p) p^{\nu}} \sum_{j=1}^{h(-q)} \psi\left(Q_{j}\right) \Theta_{\nu}\left(Z, Q_{j}, \chi\right) \circ \Pi(p) .
$$

By Lemma 14, this is equal to

$$
\begin{gathered}
\frac{p+\chi_{Q}(p)}{\chi(p) p^{\nu}} \sum_{j=1}^{h(-q)} \psi\left(Q_{j}\right)\left(\Theta_{\nu}\left(Z, Q_{j} \times \mathfrak{p}, \chi\right)+\Theta_{\nu}\left(Z, Q_{j} \times \overline{\mathfrak{p}}, \chi\right)\right) \\
=\frac{p+\chi_{Q}(p)}{\chi(p) p^{\nu}}\left(\psi(\overline{\mathfrak{p}}) \sum_{j=1}^{h(-q)} \psi\left(Q_{j} \times \mathfrak{p}\right) \Theta_{\nu}\left(Z, Q_{j} \times \mathfrak{p}, \chi\right)\right. \\
\left.+\psi(\mathfrak{p}) \sum_{j=1}^{h(-q)} \psi\left(Q_{j} \times \overline{\mathfrak{p}}\right) \Theta_{\nu}\left(Z, Q_{j} \times \overline{\mathfrak{p}}, \chi\right)\right) \\
=\frac{p+1}{\chi(p) p^{\nu}}(\psi(\mathfrak{p})+\psi(\overline{\mathfrak{p}})) \sum_{j=1}^{h(-q)} \psi\left(Q_{j}\right) \Theta_{\nu}\left(Z, Q_{j}, \chi\right)
\end{gathered}
$$

if $\chi_{Q}(p)=1$, and to 0 if $\chi_{Q}(p)=-1$. This proves formulas (4.9).

Now, let $T=\eta\left(T_{1}^{2}\left(p^{2}\right)\right)$. Then, using Proposition 8 and the notation (4.12), we obtain

$$
\begin{aligned}
F \|_{\nu+1} T=\frac{1}{\chi\left(p^{2}\right) p^{2 \nu}}\left(\left(\chi_{Q}(p)(p+1)\right.\right. & \left.+p^{-2}\left(\chi_{Q}(p) p-1\right)\right) \sum_{j=1}^{h(-q)} \psi\left(Q_{j}\right) \Theta_{\nu}\left(Z, Q_{j}, \chi\right) \\
& \left.+\chi_{Q}(p) \sum_{j=1}^{h(-q)} \psi\left(Q_{j}\right) \Theta_{\nu}\left(Z, Q_{j}, \chi\right) \circ \Pi\left(p^{2}\right)\right) .
\end{aligned}
$$

If $\chi_{Q}(p)=1$, then, by Lemma 14 , this is equal to

$$
\begin{gathered}
\frac{1}{\chi\left(p^{2}\right) p^{2 \nu}}\left(\left(p+1+p^{-1}-p^{-2}\right) \sum_{j=1}^{h(-q)} \psi\left(Q_{j}\right) \Theta_{\nu}\left(Z, Q_{j}, \chi\right)\right. \\
\left.\quad+\sum_{j=1}^{h(-q)} \psi\left(Q_{j}\right)\left(\Theta_{\nu}\left(Z, Q_{j} \times \mathfrak{p}^{2}, \chi\right)+\Theta_{\nu}\left(Z, Q_{j} \times \overline{\mathfrak{p}}^{2}, \chi\right)\right)\right) \\
=\frac{1}{\chi(p)^{2} p^{2(\nu+1)}}\left(p^{3}+p^{2}+p-1+(p \psi(\mathfrak{p}))^{2}+(p \psi(\overline{\mathfrak{p}}))^{2}\right) F .
\end{gathered}
$$

But if $\chi_{Q}(p)=-1$, then

$$
F \|_{\nu+1} T=\frac{-\left(p^{3}+p^{2}+p+1\right)}{\chi(p)^{2} p^{2(\nu+1)}} F .
$$

This proves formula (4.10).

Finally, let $T=\eta\left(T_{2}^{2}\left(p^{2}\right)\right)$. By Proposition 8 , or directly by the definition of the Hecke operators, we obtain

$$
F \|_{\nu+1} T=\frac{1}{\chi\left(p^{2}\right) p^{2 \nu}} \sum_{j=1}^{h(-q)} \psi\left(Q_{j}\right) p^{-2} \Theta_{\nu}\left(Z, Q_{j}, \chi\right)=\frac{1}{\chi\left(p^{2}\right) p^{2(\nu+1)}} F,
$$

which proves formula (4.11) and the theorem. 


\section{§5. Zeta FUnCtions of MAASs Theta SERIES OF BINARY FORMS}

Let $F \in \mathfrak{M}_{k}\left(\Gamma^{2}(q)\right)$ be a nonzero eigenfunction for all Hecke operators of the regular Hecke-Shimura ring $\mathcal{H}^{2}(q)$ :

$$
F \|_{k} T=\lambda_{F}(T) F \quad\left(\forall T \in \mathcal{H}^{2}(q)\right) .
$$

As was noted in $\S 3$, the $\operatorname{ring} \mathcal{H}^{2}(q)$ is generated over $\mathbb{C}$ by the elements (3.14) for all prime numbers $p$ not dividing $q$. For any such $p$, we introduce the p-polynomial of the eigenfunction $F$ by

$$
\begin{aligned}
\mathcal{Q}_{p, F}(t)=1 & -p^{2 k-3} \lambda_{F}(\widetilde{T}(p)) t+p^{4 k-6} \lambda_{F}\left(p \widetilde{T}_{1}\left(p^{2}\right)+p\left(p^{2}+1\right) \widetilde{T}_{2}\left(p^{2}\right)\right) t^{2} \\
& -p^{6 k-6} \lambda_{F}\left(\widetilde{T}(p) \widetilde{T}_{2}\left(p^{2}\right)\right) t^{3}+p^{8 k-6} \lambda_{F}\left(\widetilde{T}_{2}\left(p^{2}\right)^{2}\right) t^{4}
\end{aligned}
$$

and define the zeta function of $F$ as the Euler product

$$
Z_{F}(s)=\prod_{p \nmid q} \mathcal{Q}_{p, F}\left(p^{-s}\right)^{-1}
$$

(compare with [1, §1.3] and [2, §4.3.2]). It can be shown that the product converges absolutely and uniformly in some right half-plane of the complex variable $s$.

Theorem 15. Let $-q=d l^{2}$ be the discriminant of an even primitive positive definite matrix $Q$ of order 2, where $d$ is the discriminant of the field $\mathbf{K}=\mathbb{Q}(\sqrt{-q})$, and let $Q_{1}, \ldots, Q_{h(-q)}$ be a system of representatives of all different proper equivalence classes (3.17) of even primitive positive definite matrices of order 2 and discriminant $-q$. Finally, let $\psi$ be a character of the group $H(-q)$ of the proper equivalence classes under the composition (4.6), let $g$ be a rational integer, and let $\chi$ be a Dirichlet character modulo g. Suppose that the linear combination

$$
F=\Theta_{\nu}(Z, q, \psi, \chi)=\sum_{j=1}^{h(-q)} \psi\left(Q_{j}\right) \Theta_{\nu}\left(Z, Q_{j}, \chi\right) \quad(\nu=0,1)
$$

of Maass theta series is not identically zero. Then the zeta function (5.2) of the eigenfunction $F$ satisfies the identity

$$
Z_{F}(s)=L_{\mathcal{O}_{l}}(s-\nu, \psi, \bar{\chi}) L_{\mathcal{O}_{l}}(s-\nu+1, \psi, \bar{\chi}),
$$

where $\mathcal{O}_{l}$ is the subring of index $l$ in the maximal order of $\mathbf{K}$, and where

$$
L_{\mathcal{O}_{l}}(s, \psi, \bar{\chi})=\prod_{\mathfrak{p}}\left(1-\frac{\psi(\mathfrak{p}) \bar{\chi}(N(\mathfrak{p}))}{N(\mathfrak{p})^{s}}\right)^{-1}
$$

(with $\mathfrak{p}$ running over all regular prime ideals of the ring $\mathcal{O}_{l}$ not dividing $g q$ ) is an $L$ function of $\mathcal{O}_{l}$.

Proof. By Theorem 12, the function $F$ is a modular form of weight $k=\nu+1$ and character $\chi_{Q}$ for the group $\Gamma_{0}^{2}\left(q^{\prime}\right)$ of level $q^{\prime}=q g^{2}$, and $F$ is an eigenfunction for all Hecke operators corresponding to the elements (3.24) for all prime numbers $p \nmid q^{\prime}$ with eigenvalues given by (4.9)-(4.11). Substituting the eigenvalues to the formulas for the coefficients of the polynomials (5.1), we see that if $\chi_{Q}(p)=1$, then

$$
\begin{aligned}
\mathcal{Q}_{p, F}(t)= & -p^{\nu-1}(p+1)(\psi(\mathfrak{p})+\psi(\overline{\mathfrak{p}}) \bar{\chi}(p) t \\
& +p^{2 \nu-3}\left(p^{3}+p^{2}+p-1+p^{2} \psi(\mathfrak{p})^{2}+p^{2} \psi(\overline{\mathfrak{p}})^{2}+p^{2}+1\right) \bar{\chi}(p)^{2} t^{2} \\
& \quad-p^{3 \nu-2}(p+1)(\psi(\mathfrak{p})+\psi(\overline{\mathfrak{p}})) \bar{\chi}(p)^{3} t^{3}+p^{4 \nu-2} \bar{\chi}(p)^{4} t^{4} \\
= & \left(1-p^{\nu} \psi(\mathfrak{p}) \bar{\chi}(p) t\right)\left(1-p^{\nu} \psi(\overline{\mathfrak{p}}) \bar{\chi}(p) t\right)\left(1-p^{\nu-1} \psi(\mathfrak{p}) \bar{\chi}(p) t\right)\left(1-p^{\nu-1} \psi(\overline{\mathfrak{p}}) \bar{\chi}(p) t\right),
\end{aligned}
$$


but if $\chi_{Q}(p)=-1$, then

$$
\begin{aligned}
\mathcal{Q}_{p, F}(t) & =1+p^{2 \nu-3}\left(-p^{3}-p^{2}-p-1+p^{2}+1\right) \bar{\chi}(p)^{2} t^{2}+p^{4 \nu-2} \bar{\chi}(p)^{4} t^{4} \\
& =\left(1-p^{2 \nu} \bar{\chi}\left(p^{2}\right) t^{2}\right)\left(1-p^{2 \nu-2} \bar{\chi}\left(p^{2}\right) t^{2}\right) .
\end{aligned}
$$

Since, by Proposition 10, in the first case $\mathfrak{p}$ and $\overline{\mathfrak{p}}$ are the only regular prime ideals of the ring $\mathcal{O}_{l}$ of the norm $N(\mathfrak{p})=N(\overline{\mathfrak{p}})=p$, and in the second case the principal ideal $\mathfrak{p}=p \mathcal{O}_{l}$ is the only regular prime ideal of the norm $N(\mathfrak{p})=p^{2}$, the above calculations and the factorization (5.4) show that the infinite products on both sides of (5.3) have equal $p$-factors for every prime number $p$ not dividing $q^{\prime}$.

\section{REFERENCES}

[1] A. N. Andrianov, Euler products that correspond to Siegel's modular forms of genus 2, Uspekhi Mat. Nauk 29 (1974), no. 3, 43-110; English transl., Russian Math. Surveys 29 (1974), no. 3, 45-116. MR0432552 (55:5540)

[2] _ Quadratic forms and Hecke operators, Grundlehren Math. Wiss., Bd. 286, Springer-Verlag, Berlin, 1987. MR0884891 (88g:11028)

[3] — Composition of solutions of quadratic Diophantine equations, Uspekhi Mat. Nauk 46 (1991), no. 2, 3-40; English transl., Russian Math. Surveys 46 (1991), no. 2, 1-44. MR1125271 (93e:11049)

[4] , Factorizations of integral representations of binary quadratic forms, Algebra i Analiz 5 (1993), no. 1, 81-108; English transl., St. Petersburg Math. J. 5 (1994), no. 1, 71-95. MR1220490 (94h:11034)

[5] - Symmetries of harmonic theta functions of integer-valued quadratic forms, Uspekhi Mat. Nauk 50 (1995), no. 4(304), 3-44; English transl., Russian Math. Surveys 50 (1995), no. 4, 661-700. MR1357882(96i:11041)

[6] - Harmonic theta functions and Hecke operators, Algebra i Analiz 8 (1996), no. 5, 1-31; English transl., St. Petersburg Math. J. 8 (1997), no. 5, 695-720. MR1428987(98a:11053)

[7] E. Freitag, Die Wirkung von Heckeoperatoren auf Thetareihen mit harmonischen Koeffizienten, Math. Ann. 258 (1981/82), 419-440. MR0650947 (83h:10049)

[8] _ Singular modular forms and theta relations, Lecture Notes in Math., vol. 1487, SpringerVerlag, Berlin, 1991. MR1165941 (94b:11038)

[9] H. Maass, Konstruktion von Spitzenformen beliebigen Grades mit Hilfe von Thetareihen, Math. Ann. 226 (1977), 275-284. MR0444575 (56:2925)

[10] R. Salvati-Manni and J. Top, Cusp forms of weight 2 for the group $\Gamma_{2}(4,8)$, Amer. J. Math. 115 (1993), no. 2, 455-486. MR 1216438 (94e:11050)

[11] H. Yoshida, Siegel's modular forms and the arithmetic of quadratic forms, Invent. Math. 60 (1980), no. 3, 193-248. MR0586427(81m:10051)

St. Petersburg Branch, Steklov Mathematical Institute, Russian Academy of Sciences, Fontanka 27, St. Petersburg 191023, Russia

E-mail address: anandr@pdmi.ras.ru, anatoli.andrianov@gmail.com

Received 5/APR/2007

Translated by THE AUTHOR 